\title{
Best constants for the isoperimetric inequality in quantitative form
}

Received February 17, 2011 and in revised form October 19, 2011

\begin{abstract}
We prove some results on isoperimetric inequalities with quantitative terms. In the 2-dimensional case, our main contribution is a method for determining the optimal coefficients $c_{1}, \ldots, c_{m}$ in the inequality $\delta P(E) \geq \sum_{k=1}^{m} c_{k} \alpha(E)^{k}+o\left(\alpha(E)^{m}\right)$, valid for each Borel set $E$ with positive and finite area, with $\delta P(E)$ and $\alpha(E)$ being, respectively, the isoperimetric deficit and the Fraenkel asymmetry of $E$. In $n$ dimensions, besides proving existence and regularity properties of minimizers for a wide class of quantitative isoperimetric quotients including the lower semicontinuous extension of $\delta P(E) / \alpha(E)^{2}$, we describe a general technique upon which our 2-dimensional result is based. This technique, called Iterative Selection Principle, extends the one introduced in [12].
\end{abstract}

Keywords. Best constants, isoperimetric inequality, quasiminimizers of the perimeter

\section{Introduction}

Given $n \geq 2$, let $\mathcal{S}^{n}$ be the collection of all Borel sets $E \subset \mathbb{R}^{n}$ with positive and finite Lebesgue measure $|E|$. Denoting by $B_{E}$ the open ball centered at 0 with the same measure as $E$ and by $P(E)$ the perimeter of $E$ in the sense of De Giorgi, the isoperimetric deficit and the Fraenkel asymmetry index of $E \in \mathcal{S}^{n}$ are, respectively,

$$
\delta P(E)=\frac{P(E)-P\left(B_{E}\right)}{P\left(B_{E}\right)}
$$

and

$$
\alpha(E)=\inf \left\{\left|E \triangle\left(x+B_{E}\right)\right| /\left|B_{E}\right|: x \in \mathbb{R}^{n}\right\},
$$

where, as usual, $V \triangle W$ denotes the symmetric difference of the two sets $V$ and $W$.

The sharp quantitative isoperimetric inequality can be stated as follows: there exists a constant $C=C(n)>0$ such that

$$
\delta P(E) \geq C \alpha(E)^{2} .
$$

M. Cicalese: Technische Universität München, Zentrum Mathematik - M7, Boltzmannstraße 3, 85747 Garching, Germany; e-mail: cicalese@ma.tum.de

G. P. Leonardi: Dipartimento di Scienze Fisiche, Informatiche e Matematiche,

Università degli Studi di Modena e Reggio Emilia, Via Campi 213/b, I-41100 Modena, Italy;

e-mail: gianpaolo.leonardi@unimore.it

Mathematics Subject Classification (2010): Primary 52A40; Secondary 28A75, 49J45 
In recent years, after some partial results on quantitative isoperimetric and Sobolev inequalities (see [14] and [10]) and starting from the general proof of the sharp quantitative isoperimetric inequality by Fusco, Maggi and Pratelli [17] (see also [15] and [12] for different proofs), great effort has been dedicated to proving quantitative versions of several analytic-geometric inequalities. We recall in particular [16], [18], [8], [9], [19], [20] (see [25] for a survey on this topic). However, some relevant issues-such as the determination of the best constant in (1.2), that is, of

$$
C_{\text {best }}:=\max \left\{C>0: \delta P(E) \geq C \alpha(E)^{2}, \forall E \in \mathcal{S}^{n}\right\}
$$

the regularity of the optimal set $E_{\text {best }}$ such that $C_{\text {best }}=\delta P\left(E_{\text {best }}\right) / \alpha\left(E_{\text {best }}\right)^{2}$, as well as the shape of such a set-have not yet been considered in their full generality. They seem to be challenging problems and only few results are known. This is basically due to the presence of the Fraenkel asymmetry index which makes (1.3) a nonlocal problem. As a consequence, (1.3) is difficult to tackle via standard arguments of calculus of Variations and shape optimization.

Only in dimension $n=2$, but within the class of convex sets, are the minimizers of the isoperimetric deficit (i.e., of the perimeter) at a fixed asymmetry index known explicitly. Indeed, in 1992 Campi proved ([7, Theorem 4]) the following equivalent statement: among all convex sets $E \in \mathcal{S}^{2}$ with fixed area and perimeter $P(E)=\sigma$, there exists a unique set $E_{\sigma}$ that maximizes the Fraenkel asymmetry. This obviously entails existence and uniqueness in (1.3) restricted to convex sets. It moreover implies that the optimal convex set $E_{\text {conv }}$ agrees with $E_{\sigma}$ for a suitable $\sigma$.

By exploiting a symmetrization technique due to Bonnesen [5], also known as annular symmetrization, Campi completely characterized the set $E_{\sigma}$ and found an explicit threshold $\sigma_{0}$ such that, depending on whether $\sigma$ is above or below $\sigma_{0}, E_{\sigma}$ is either what he called an oval, or a biscuit. Here, following Campi's definition, and assuming without loss of generality that the Fraenkel asymmetry of $E$ is realized at $x=0$ (that is, $B_{E}$ is an optimal ball for $E$ in the sense that $\left.\alpha(E)=\left|E \triangle B_{E}\right| /\left|B_{E}\right|\right)$ we define an oval to be a set whose boundary is composed of two pairs of equal and opposite circular arcs, with endpoints on $\partial B_{E}$ and with common tangent lines at each point, while a biscuit is a set which is obtained by capping a rectangle with two half disks (see Figure 1). In the recent paper [2], the authors, besides proving Campi's result in a slightly different way, optimize the quotient $\delta P\left(E_{\sigma}\right) / \alpha\left(E_{\sigma}\right)^{2}$ to find that $C_{\mathrm{conv}}=\min _{\sigma} \delta P\left(E_{\sigma}\right) / \alpha\left(E_{\sigma}\right)^{2} \simeq 0.405585$ and that $E_{\text {conv }}$ is a biscuit.
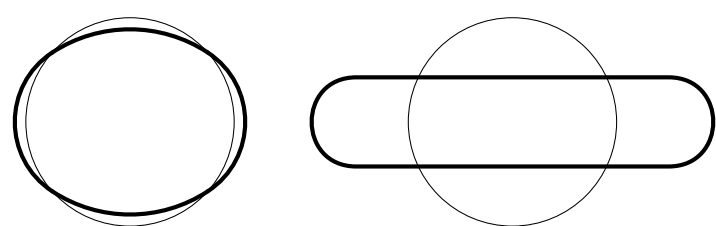

Fig. 1. An oval and a biscuit, together with their optimal balls. 
However, it is worth noting that, in dimension $n=2$, the problem (1.3) is not solved by a convex set. An example of a nonconvex set $E_{\mathrm{nc}}$ for which

$$
\frac{\delta P\left(E_{\mathrm{nc}}\right)}{\alpha\left(E_{\mathrm{nc}}\right)^{2}} \simeq 0.39314
$$

is provided by the mask, a set with two orthogonal axes of symmetry and with only two optimal balls, whose boundary is made up of eight suitable circular arcs (see Figure 2). In the forthcoming paper [11] it will be proved that such a set realizes the best constant within a quite rich subclass of planar sets. Therefore, it may seem reasonable to conjecture that the mask is optimal with respect to all sets in $\mathbb{R}^{2}$. Up to our knowledge, apart from some partial results in the two-dimensional case, problem (1.3) is widely open.

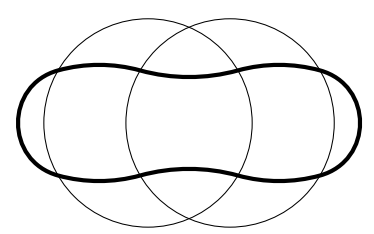

Fig. 2. The mask, with its two optimal balls.

Another relevant issue is the determination of the optimal coefficients $c_{1}, \ldots, c_{m}$ in the asymptotic estimate

$$
\delta P(E) \geq \sum_{k=1}^{m} c_{k} \alpha(E)^{k}+o\left(\alpha(E)^{m}\right) .
$$

This problem was originally raised by Hall, Hayman and Weitsman [24], who conjectured that

$$
\delta P(E) \geq \frac{\pi}{8(4-\pi)} \alpha(E)^{2}+o\left(\alpha(E)^{2}\right)
$$

for any convex set $E \subset \mathbb{R}^{2}$ with finite positive area, and that the constant $\pi /(8(4-\pi))$ is optimal. The positive answer to this conjecture was given by Hall and Hayman [23]. We observe, however, that the conjecture can also be proved via Campi's result (see [7] and Section 3 for more details).

In [22] Hall conjectured that the inequality (1.5) should hold even without the convexity assumption. In [12] we have proved this and announced a more refined result in this direction, which we present here.

It is worth pointing out that (1.5) is an easy consequence of the explicit determination of minimizers of perimeter at a fixed (small) asymmetry. By Campi's result, we know that the minimizers among convex sets with small asymmetry are necessarily ovals. With this information in the convex, 2-dimensional case, it is possible to prove not only (1.5) but also a whole family of lower bounds of the isoperimetric deficit by a certain polynomial in the asymmetry plus higher-order terms as in (1.4) (see Remark 2.1 in [2]). 
Our main result is Theorem 3.2, in which we show that even the refined estimate (1.4) still holds for any set $E \in \mathcal{S}^{2}$ and not only for convex sets, with optimal coefficients $c_{1}, \ldots, c_{m}$ being as in the convex case. To better explain this point, we introduce the following family of functionals: for any $E \in \mathcal{S}^{2}$ we define

$$
Q^{(1)}(E)= \begin{cases}\frac{\delta P(E)}{\alpha(E)} & \text { if } \alpha(E)>0 \\ \inf \left\{\liminf _{h} Q^{(1)}\left(E_{h}\right): \alpha\left(E_{h}\right)>0,\left|E_{h} \Delta B\right| \rightarrow 0\right\} & \text { otherwise }\end{cases}
$$

and, for a given integer $m \geq 2$ and assuming that $Q^{(m-1)}(B) \in \mathbb{R}$, we set

$$
Q^{(m)}(E)= \begin{cases}\frac{Q^{(m-1)}(E)-Q^{(m-1)}(B)}{\alpha(E)} & \text { if } \alpha(E)>0, \\ \inf \left\{\liminf _{h} Q^{(m)}\left(E_{h}\right): \alpha\left(E_{h}\right)>0,\left|E_{h} \Delta B\right| \underset{h}{\rightarrow} 0\right\} & \text { otherwise. }\end{cases}
$$

It is easy to check that, by definition, $c_{k}=Q^{(k)}(B)$, hence the problem of finding the optimal coefficients in (1.4) is reduced to the computation of $Q^{(k)}(B)$. Combining the existence and regularity results proved in Section 4 with a penalization technique analogous to the one exploited in [12], we derive the following result (see Theorem 3.2 and Proposition 3.3):

Best Asymptotic Constants in the Plane. Given an integer $m \geq 1$, the estimate (1.4) is satisfied for any Borel set $E \subset \mathbb{R}^{2}$ with $0<|E|<\infty$, with optimal constants

$$
c_{k}=Q^{(k)}(B)=\lim _{j} Q^{(k)}\left(E_{j}^{(k)}\right)
$$

and where $\left(E_{j}^{(k)}\right)_{j}$ is a sequence of ovals converging to B. Moreover, $c_{k}$ is computable since both the perimeter and the asymmetry of ovals are explicit functions of a real variable.

We shall give a proof of this result and show some optimal coefficients in Section 3. One of the main tools we use in the proof is the Iterative Selection Principle, which is an iterative version of the method we developed in [12]. In Section 5 we detail this technique in the general case of dimension $n \geq 2$. Here we specialize it to the 2-dimensional case:

Iterative Selection Principle $(n=2)$. Let $m \geq 2$ and assume that $Q^{(k)}(B) \in \mathbb{R}$ for all $k=1, \ldots, m-1$. Then there exists a sequence $\left(E_{j}^{(m)}\right)_{j} \subset \mathcal{S}^{2}$ of sets such that

(i) $\left|E_{j}^{(m)}\right|=|B|, \alpha\left(E_{j}^{(m)}\right)>0$ and $\alpha\left(E_{j}^{(m)}\right) \rightarrow 0$ as $j \rightarrow \infty$;

(ii) $Q^{(m)}\left(E_{j}^{(m)}\right) \rightarrow Q^{(m)}(B)$ as $j \rightarrow \infty$ and $E_{j}^{(m)}$ minimizes $Q^{(m)}$ among all sets $F$ with $\alpha(F)=\alpha\left(E_{j}^{(m)}\right)$; 
(iii) for each $j$ there exists a function $u_{j}^{(m)} \in C^{1,1}(\partial B)$ such that

$$
\partial E_{j}^{(m)}=\left\{\left(1+u_{j}^{(m)}(x)\right) x: x \in \partial B\right\}
$$

and $u_{j}^{(m)} \rightarrow 0$ in the $C^{1}$-norm as $j \rightarrow \infty$;

(iv) $\partial E_{j}^{(m)}$ has weak curvature $\kappa_{j}^{(m)} \in L^{\infty}\left(\partial E_{j}^{(m)}\right)$ and $\left\|\kappa_{j}^{(m)}-1\right\|_{L^{\infty}\left(\partial E_{j}^{(m)}\right)} \rightarrow 0$ as $j \rightarrow \infty$; moreover, $\kappa_{j}^{(m)}$ is constant in any free region ${ }^{1}$ for $E_{j}^{(m)}$.

In Section 3 we will prove that, thanks to the Iterative Selection Principle, $E_{j}^{(m)}$ must belong to a very special class of convex sets, whose boundary is made up of arcs with only two different curvatures. Finally, by applying Bonnesen's annular symmetrization to sets belonging to this class, we are able to conclude in a very elementary way that $E_{j}^{(m)}$ is necessarily an oval converging to $B$ as $j \rightarrow \infty$.

In the last two sections we collect our main technical tools, and prove them in the general, $n$-dimensional case for future reference. In particular in Section 4 we show existence and regularity results for a wide class of quantitative isoperimetric quotients. More specifically, given two Lipschitz-continuous functions $f, g:[0,2] \rightarrow \mathbb{R}$ with $g(t)$ nonnegative and zero if and only if $t=0$, for all $E \in \mathcal{S}^{n}$ we define the functional

$$
\mathcal{F}_{f, g}(E)= \begin{cases}\frac{\delta P(E)+f(\alpha(E))}{g(\alpha(E))} & \text { if } \alpha(E)>0, \\ \inf \left\{\liminf _{h} \mathcal{F}_{f, g}\left(E_{h}\right): \alpha\left(E_{h}\right)>0,\left|E_{h} \Delta B\right| \rightarrow 0\right\} & \text { otherwise, }\end{cases}
$$

and for all $\alpha_{0}>0$ we consider the minimum problem

$$
\min \left\{\mathcal{F}_{f, g}(E): E \in \mathcal{S}^{n}, \alpha(E) \geq \alpha_{0}\right\} .
$$

In Theorem 4.1 we prove that (1.6) has a solution, while in Theorems 4.3 and 4.4 we prove that the minima are actually $\Lambda$-minimizers of perimeter (see Section 2 for the definition). As a consequence, by classical results in the regularity theory for quasiminimizers of perimeter (see Theorem 2.1), these minima are of class $C^{1, \gamma}$ for all $\gamma<1$ (and of class $C^{1,1}$ in dimension $n=2$ ). Note that, by choosing $f=0$ and $g(t)=t^{2}$, we have $\mathcal{F}_{f, g}(E)=\delta P(E) / \alpha(E)^{2}$, hence the existence and regularity statements hold in particular for problem (1.3).

Finally, in Section 5 we prove the following $n$-dimensional version of our Iterative Selection Principle (see Theorem 5.1):

Iterative Selection Principle $(n \geq 2)$. Let $m \geq 2$ and assume that $Q^{(k)}(B) \in \mathbb{R}$ for all $k=1, \ldots, m-1$. Then there exists a sequence $\left(E_{j}^{(m)}\right)_{j} \subset \mathcal{S}^{n}$ of sets such that

(i) $\left|E_{j}^{(m)}\right|=|B|, \alpha\left(E_{j}^{(m)}\right)>0$ and $\alpha\left(E_{j}^{(m)}\right) \rightarrow 0$ as $j \rightarrow \infty$;

(ii) $Q^{(m)}\left(E_{j}^{(m)}\right) \rightarrow Q^{(m)}(B)$ as $j \rightarrow \infty$;

\footnotetext{
1 See Section 2 for the precise definition of free region.
} 
(iii) for each $j$ there exists a function $u_{j}^{(m)} \in C^{1, \gamma}(\partial B)$, for all $0<\gamma<1$, such that

$$
\partial E_{j}^{(m)}=\left\{\left(1+u_{j}^{(m)}(x)\right) x: x \in \partial B\right\}
$$

and $u_{j}^{(m)} \rightarrow 0$ in the $C^{1}$-norm as $j \rightarrow \infty$;

(iv) $\partial E_{j}^{(m)}$ has weak mean curvature $H_{j}^{(m)} \in L^{\infty}\left(\partial E_{j}^{(m)}\right)$ and

$$
\left\|H_{j}^{(m)}-1\right\|_{L^{\infty}\left(\partial E_{j}^{(m)}\right)} \rightarrow 0 \quad \text { as } j \rightarrow \infty .
$$

Moreover, $H_{j}^{(m)}$ is constant on any free region for $E_{j}^{(m)}$.

In analogy with [12], the main idea for proving Theorem 5.1 is to combine the results of Section 4 with a suitable penalization technique, even though some extra, technical difficulty arises, as we cannot a priori assume that $Q^{(m)}(B)$ is finite.

\section{Notation and preliminaries}

Let $E \subset \mathbb{R}^{n}$ be a Borel set, with $n$-dimensional Lebesgue measure $|E|$. Given $x \in \mathbb{R}^{n}$ and $r>0$, we denote by $B(x, r)$ the open Euclidean ball with center $x$ and radius $r$. We also set $B=B(0,1)$ and $\omega_{n}=|B|$. For a set $E \subset \mathbb{R}^{n}$ we denote by $\chi_{E}$ its characteristic function and define the $L^{1}$ (or $L_{\text {loc }}^{1}$ ) convergence of a sequence of sets to a limit set in terms of the $L^{1}$ (or $L_{\text {loc }}^{1}$ ) convergence of their characteristic functions.

The perimeter of a Borel set $E$ inside an open set $\Omega \subset \mathbb{R}^{n}$ is

$$
P(E, \Omega):=\sup \left\{\int_{E} \operatorname{div} g(x) d x: g \in C_{c}^{1}\left(\Omega ; \mathbb{R}^{n}\right),|g| \leq 1\right\} .
$$

By Gauss-Green's Theorem, this provides an extension of the Euclidean, $(n-1)$-dimensional measure of a smooth (or Lipschitz) boundary $\partial E$. We will simply write $P(E)$ instead of $P\left(E, \mathbb{R}^{n}\right)$, and we will say that $E$ is a set of finite perimeter if $P(E)<\infty$. One can check that $P(E, \Omega)<\infty$ if and only if the distributional derivative $D \chi_{E}$ is a vectorvalued Radon measure in $\Omega$ with finite total variation $\left|D \chi_{E}\right|(\Omega)$. By known results (see e.g. [4]) one has $D \chi_{E}=v_{E} \mathcal{H}^{n-1}\left\lfloor\partial^{*} E\right.$ where $\mathcal{H}^{n-1}$ is the $(n-1)$-dimensional Hausdorff measure and $\partial^{*} E$ is the reduced boundary of $E$, i.e., the set of those points $x \in \partial E$ such that the generalized inner normal $v_{E}(x)$ is defined, where

$$
v_{E}(x)=\lim _{r \rightarrow 0} \frac{D \chi_{E}(B(x, r))}{\left|D \chi_{E}\right|(B(x, r))} \quad \text { and } \quad\left|v_{E}(x)\right|=1 .
$$

We say that a set $E \subset \mathbb{R}^{n}$ of locally finite perimeter is a strong $\Lambda$-minimizer of perimeter (here, we adopt the terminology used in [3]) if there exists $R>0$ such that, for all $x \in \mathbb{R}^{n}$ and $0<r<R$, and for any compact variation $F$ of $E$ in $B(x, r)$ (that is, $E \triangle F \subset \subset B(x, r))$ one has

$$
P(E, B(x, r)) \leq P(F, B(x, r))+\Lambda|E \Delta F| .
$$


We shall then write $E \in \mathcal{Q} \mathcal{M}(R, \Lambda)$ to underline the dependence of the definition of strong $\Lambda$-minimality on the parameters $R$ and $\Lambda$.

Strong $\Lambda$-minimizers and more generally quasiminimizers of perimeter have been studied since the seminal work [13] by De Giorgi on the regularity theory for minimal surfaces. We also mention the paper by Massari [26] on the regularity of boundaries with prescribed mean curvature (i.e., of minimizers of the functional $P(E)+\int_{E} h(x) d x$ ), and the clear, as well as general, analysis of the regularity of quasiminimizers of perimeter due to Tamanini [27, 28] and the lecture notes [3] by Ambrosio. It is worth mentioning that a further (substantial) extension of the regularity theory for quasiminimizers in the context of currents and varifolds is due to Almgren [1].

In the following theorem we state three crucial properties of uniform sequences of $\Lambda$-minimizers that converge in $L_{\text {loc }}^{1}$ to some limit set $F$. These properties can be derived from results contained for instance in [28] and [3] (see also [12] for more details).

Theorem 2.1. Let $E_{1}, \ldots, E_{h}, \ldots$ belong to $\mathcal{Q M}(R, \Lambda)$ for some fixed $R, \Lambda>0$ and let $E_{h}$ converge to a Borel set $F$ in $L_{\text {loc }}^{1}\left(\mathbb{R}^{n}\right)$ as $h \rightarrow \infty$. Then the following facts hold:

(i) $F \in \mathcal{Q M}(R, \Lambda)$. Moreover, if $\partial F$ is bounded then $\partial E_{h}$ converges to $\partial F$ in the Hausdorff metric. ${ }^{2}$

(ii) $\partial^{*} F$ is a smooth, $(n-1)$-dimensional hypersurface of class $C^{1, \gamma}$ for all $\gamma \in(0,1)$ (and $C^{1,1}$ in dimension $n=2$ ), while the singular set $\partial F \backslash \partial^{*} F$ has Hausdorff dimension $\leq n-8$.

(iii) If $\partial F$ is smooth (i.e., if the singular set of $\partial F$ is empty) then there exists $h_{0}$ such that, for any $h \geq h_{0}, \partial E_{h}$ has no singular points, and thus it is of class $C^{1, \gamma}$ for all $0<\gamma<1\left(C^{1,1}\right.$ if $\left.n=2\right)$. Moreover, if $\partial F$ is compact then $\partial E_{h}$ can be represented as the normal graph of a smooth function $u_{h}$ defined on $\partial F$ and such that $u_{h} \rightarrow 0$ in $C^{1}(\partial F)$ as $h \rightarrow \infty$.

We will denote by $\mathcal{S}^{n}$ the class of Borel subsets of $\mathbb{R}^{n}$ with finite positive Lebesgue measure. Moreover, given $0<\beta<1$ we set

$$
\mathcal{S}_{\beta}^{n}:=\left\{E \subset \mathcal{S}^{n}:|E|=|B|, \alpha(E) \geq \beta\right\} .
$$

Given $E \in \mathcal{S}^{n}$ we define its isoperimetric deficit $\delta P(E)$ and its Fraenkel asymmetry $\alpha(E)$ as follows:

$$
\begin{aligned}
\delta P(E) & :=\frac{P(E)-P\left(B_{E}\right)}{P\left(B_{E}\right)}, \\
\alpha(E) & :=\inf \left\{\frac{\left|E \triangle\left(x+B_{E}\right)\right|}{\left|B_{E}\right|}: x \in \mathbb{R}^{n}\right\},
\end{aligned}
$$

where $B_{E}$ denotes the ball centered at the origin such that $\left|B_{E}\right|=|E|$ and $\triangle$ denotes symmetric difference. Since both $\delta P(E)$ and $\alpha(E)$ are invariant under isometries and

\footnotetext{
2 A sequence of compact sets $K_{h}$ converges to a compact set $K$ in the Hausdorff metric iff the infimum of all $\varepsilon>0$ such that $K \subset K_{h}+\varepsilon B$ and $K_{h} \subset K+\varepsilon B$ (i.e., the so-called Hausdorff distance between $K_{h}$ and $K$ ) tends to 0 as $h \rightarrow \infty$.
} 
dilations, from now on we will set $|E|=|B|$ so that $B_{E}=B$. By definition, the Fraenkel asymmetry $\alpha(E)$ satisfies $\alpha(E) \in[0,2)$ and it is zero if and only if $E$ coincides with $B$ in measure-theoretic sense and up to a translation. Notice that the infimum in (2.2) is actually a minimum.

We will denote by $\mathcal{Z}(E)$ the set of optimal centers for $E$, i.e. of those points $z \in \mathbb{R}^{n}$ such that $\left|E \triangle\left(z+B_{E}\right)\right|=\alpha(E)\left|B_{E}\right|$. Finally, we will say that an open set $A \subset \mathbb{R}^{n}$ is a free region for $E$ if $A$ is a connected component of

$$
\Omega=\mathbb{R}^{n} \backslash \bigcup_{z \in \mathcal{Z}(E)}(z+\partial B)
$$

Note that $\Omega$ is open, since it is the complement of a compact set.

We now introduce some useful functionals, which we call quantitative isoperimetric quotients of order $m$. For any $E \subset \mathcal{S}^{n}$ we set

$$
Q^{(1)}(E)= \begin{cases}\frac{\delta P(E)}{\alpha(E)} & \text { if } \alpha(E)>0, \\ \inf \left\{\liminf _{k} \frac{\delta P\left(F_{k}\right)}{\alpha\left(F_{k}\right)}:\left|F_{k}\right|=|B|, \alpha\left(F_{k}\right)>0,\left|F_{k} \Delta B\right| \rightarrow 0\right\} & \text { otherwise. }\end{cases}
$$

We recall that the optimal power of the asymmetry in the quantitative isoperimetric inequality is 2 , thus we necessarily have $Q^{(1)}(B)=0$. This can also be seen through a straightforward computation on a sequence of ellipses converging to the ball $B$, and similarly holds in any dimension.

For a given integer $m \geq 2$ and assuming that $Q^{(k)}(B) \in \mathbb{R}$ for all $k=1, \ldots, m-1$, we define

$$
Q^{(m)}(E)= \begin{cases}\frac{Q^{(m-1)}(E)-Q^{(m-1)}(B)}{\alpha(E)} & \text { if } \alpha(E)>0, \\ \inf \left\{\liminf _{k} Q^{(m)}\left(F_{k}\right),\left|F_{k}\right|=|B|, \alpha\left(F_{k}\right)>0,\left|F_{k} \Delta B\right| \rightarrow 0\right\} & \text { otherwise. }\end{cases}
$$

Note that, assuming $\alpha(E)>0$ and recalling that $Q^{1}(B)=0$, it turns out that

$$
Q^{(2)}(E)=\delta P(E) / \alpha(E)^{2}
$$

is precisely the sharp quantitative isoperimetric quotient. Hence, by (1.3), it is bounded from below by a positive, dimensional constant and, as a consequence, $Q^{(2)}(B)$ is finite and strictly positive.

In what follows, we shall often say that $Q^{(m)}$ is well-defined simply meaning that we are inductively assuming $Q^{(k)}(B)$ is finite for all $k=1, \ldots, m-1$. Clearly, this does not necessarily imply that also $Q^{(m)}(B)$ is finite. One can easily check that the functional $Q^{(m)}$ is lower semicontinuous with respect to $L^{1}$-convergence of sets. However, it is not possible to immediately get the finiteness of $Q^{(m)}(B)$, and in particular one cannot $a$ priori exclude that $Q^{(m)}(B)=-\infty$. 
By the previous definition, assuming $m \geq 2$ and $Q^{(m)}$ is well-defined, and $\alpha(E)>0$, one can equivalently write

$$
Q^{(m)}(E)=\frac{\delta P(E)-\psi_{m}(\alpha(E))}{\alpha(E)^{m}}
$$

where

$$
\psi_{m}(\alpha)=\sum_{k=1}^{m-1} Q^{(k)}(B) \alpha^{k}
$$

Correspondingly, for any $m \geq 2$ we define a sequence $\left(Q_{j}^{(m)}\right)_{j}$ of penalized functionals. We assume $Q^{(m-1)}(B) \in \mathbb{R}$ and choose a recovery sequence $\left(W_{j}^{(m)}\right)_{j}$ for $Q^{(m)}(B)$. Then, setting $\alpha_{j}^{(m)}=\alpha\left(W_{j}^{(m)}\right)$, for any $E \in \mathcal{S}^{n}$ with $\alpha(E)>0$ we define

$$
Q_{j}^{(m)}(E)=\frac{Q^{(m-1)}(E)-Q^{(m-1)}(B)+\left(\alpha(E) / \alpha_{j}^{(m)}-1\right)^{2}}{\alpha(E)} .
$$

One can immediately check that

$$
\begin{aligned}
Q_{j}^{(m)}(E) & =Q^{(m)}(E)+\frac{\left(\alpha(E) / \alpha_{j}^{(m)}-1\right)^{2}}{\alpha(E)} \\
& =\frac{\delta P(E)-\psi_{m}(\alpha(E))+\alpha(E)^{m-1}\left(\alpha(E) / \alpha_{j}^{(m)}-1\right)^{2}}{\alpha(E)^{m}} .
\end{aligned}
$$

Note that $Q_{j}^{(2)}$ slightly differs from the sequence of penalized functionals introduced in [12].

\section{Optimal lower bounds for the perimeter in the plane}

We present here our main 2-dimensional results. In the first one, we establish existence and regularity of minimizers of the quantitative isoperimetric quotient of order $m$ :

Theorem 3.1. Fix $m \geq 1$ and assume that $Q^{(m)}$ is well-defined. Then there exists $E^{m} \in \mathcal{S}^{2}$ such that $Q^{(m)}\left(E^{m}\right) \leq Q^{(m)}(F)$ for any $F \in \mathcal{S}^{2}$. Moreover, $\partial E^{m}$ is globally of class $C^{1,1}$ and for any free region $A$ for $E^{m}$ the set $\partial E^{m} \cap A$ is made up of arcs of constant curvature (the curvature may vary from a free region to another).

Proof. Either $Q^{(m)}(B) \leq Q^{(m)}(F)$ for all $F \in \mathcal{S}^{2}$ (and thus $B$ is the required minimizer), or inf $\mathcal{S}^{2} Q^{(m)}=\inf _{\mathcal{S}_{\beta}^{n}} Q^{(m)}$ for some $\beta>0$. In the latter case, we first observe that, on choosing $f(\alpha)=\psi_{m}(\alpha)$ and $g(\alpha)=\alpha^{m}$, we have $\mathcal{F}_{f, g}=Q^{(m)}$ (see Section 4 for the definition of $\mathcal{F}_{f, g}$ ). Then the conclusion follows by applying Theorem 4 .1, Theorem 4.4 and Remark 4.6 specialized to the case $n=2$. 
Theorem 3.1 is also valid in dimension $n>2$ : one simply has to replace " $C^{1,1}$ " with " $C^{1, \gamma}$ for all $0<\gamma<1$ " and "curvature" with "mean curvature" (see Section 4 for more details). In the case $m=2$, finding the optimal (absolute) constant $C$ in the inequality

$$
\delta P(E) \geq C \alpha(E)^{2}
$$

amounts to explicitly finding a minimizer $E$ of $Q^{(2)}$. Thanks to Theorem 3.1 one knows that $E$ exists and has some special properties. In particular, $\partial E$ is $C^{1,1}$ and its weak curvature becomes constant on any free region. Even though it is not clear how to use this result to completely identify $E$ (we recall that the infimum of $Q^{(2)}$ is not presently known) one is led to conjecture that $\partial E$ must consist of a finite number of $C^{1}$-connected arcs of circle, with possibly different curvatures. This would immediately follow if one could prove that the set of optimal centers for $E$ is necessarily finite. In this direction, we show in the forthcoming paper [11] that the minimizers of $Q^{(2)}$ on a suitably restricted class of connected, 2-symmetric sets in the plane is attained by a "mask", i.e. a 2-symmetric set having only two optimal balls, and whose boundary is made up of arcs with three different curvatures (see Figure 2). By our analysis one gets an upper bound on the optimal constant $C$ (more specifically, $C \leq 0.39314$ ).

The second theorem of this section can be regarded as the main result of the paper. Indeed, we are able to show optimal asymptotic lower bounds of the isoperimetric deficit $\delta P(E)$ in terms of polynomial functions of the asymmetry $\alpha(E)$, whose coefficients can be computed through a one-parameter family of 2-symmetric, convex deformations of the disk $B$, with boundary of class $C^{1}$ and formed by two pairs of congruent arcs of circle. These particular convex sets are known as ovals (see Figure 1 and the description below). Here is the statement of our result:

Theorem 3.2. Given an integer $m \geq 1$ we have

$$
\delta P(E) \geq \sum_{k=1}^{m} c_{k} \alpha(E)^{k}+o\left(\alpha(E)^{m}\right)
$$

for any Borel set $E \subset \mathbb{R}^{2}$ with $0<|E|<\infty$, where $c_{k}=Q^{(k)}(B)=\lim _{j} Q^{(k)}\left(E_{j}^{(k)}\right)$ is optimal and $\left(E_{j}^{(k)}\right)_{j}$ is a sequence of ovals converging to $B$.

We remark that Theorem 3.2 generalizes Theorem 4.6 in [12] as well as previous results obtained for convex sets by Hall, Hayman and Weitsman [24, 23, 22], by Campi [7] and by Alvino, Ferone and Nitsch [2].

For the reader's convenience, we describe here the family of ovals and exhibit some of the optimal constants $c_{k}$ in (3.1). Ovals are $C^{1}$ convex sets in $\mathbb{R}^{2}$ with two orthogonal axes of symmetry (hereafter the reference axes $x$ and $y$ ) and whose boundary is the union of two pairs of congruent arcs of circle. Following [2], one can give an explicit analytic expression of this family of sets $\left\{\mathcal{O}_{\xi}\right\}_{\xi \in[\arctan (\pi / 4), \pi / 4]}$ for $\left|\mathcal{O}_{\xi}\right|=|B|=\pi$, where $\xi \in[\arctan (\pi / 4), \pi / 4]$ is the angular coordinate of the unique point of intersection of $\partial \mathcal{O}_{\xi}$ and $\partial B$ in the first quadrant of the $(x, y)$-plane. Moreover it is shown in [2] that 
$\mathcal{O}_{\xi} \rightarrow \mathcal{O}_{\pi / 4}=B$ in $C^{1}$ as $\xi \rightarrow \pi / 4$ and that

$$
\begin{aligned}
\delta P\left(\mathcal{O}_{\xi}\right) & =\frac{2}{\pi}\left(\left(\frac{\sin \xi}{\cos \eta}\left(\frac{\pi}{2}-\eta\right)+\frac{\cos \xi}{\sin \eta} \eta\right)-\frac{\pi}{2}\right), \\
\alpha\left(\mathcal{O}_{\xi}\right) & =\frac{4}{\pi}\left(\frac{\sin ^{2} \xi}{\cos ^{2} \eta}\left(\frac{\pi}{2}-\eta-\sin \eta \cos \eta\right)-\xi+\sin \xi \cos \xi\right),
\end{aligned}
$$

where

$$
\tan \xi=\frac{1}{\tan \eta} \cdot \frac{\pi \sin ^{2} \eta-2 \eta+2 \sin \eta \cos \eta}{2 \eta-\pi \sin ^{2} \eta+2 \sin \eta \cos \eta}
$$

Note that the computation of $\alpha\left(\mathcal{O}_{\xi}\right)$ is made simple by the uniqueness of the optimal center of $\mathcal{O}_{\xi}$ (see also Lemma 3.5). That center coincides with the center of the coordinate system for symmetry reasons. Therefore, the statement of Theorem 3.2 can be rephrased as

Proposition 3.3. We have

$$
c_{k}=Q^{(k)}(B)=\lim _{\xi \rightarrow \pi / 4} Q^{(k)}\left(\mathcal{O}_{\xi}\right) .
$$

The exact computation of these optimal constants is elementary in principle, but extremely tedious in practice (for this reason, we have done some calculations with a basic software for symbolic calculus). Note however that $c_{2 i+1}=0$ as a consequence of the symmetry of the function $\xi \mapsto \delta P\left(\mathcal{O}_{\xi}\right) / \alpha\left(\mathcal{O}_{\xi}\right)^{2}$ with respect to $\xi=\pi / 4$. For instance, the first three nonzero coefficients are

$$
\begin{aligned}
c_{2}= & \frac{\pi}{32-8 \pi}, \\
c_{4}= & -\frac{\pi^{3}(3 \pi-14)(5 \pi-16)}{96(4-\pi)^{4}(\pi-2)}, \\
c_{6}= & \frac{-759808 \pi^{5}+1619648 \pi^{6}}{2880(4-\pi)^{7}(\pi-2)^{4}} \\
& +\frac{\pi^{7}(-1386576+\pi(612992+\pi(-148024+3(6184-315 \pi) \pi)))}{2880(4-\pi)^{7}(\pi-2)^{4}},
\end{aligned}
$$

and one has

$$
c_{2} \simeq 0.457474, \quad c_{4} \simeq-0.696215, \quad c_{6} \simeq 1.76079 .
$$

The proof of Theorem 3.2 strongly relies on the Iterative Selection Principle (see Section 5) coupled with Bonnesen's annular symmetrization (see [5]), which we describe below for the reader's convenience. Given $E \in \mathcal{S}^{2}$, we fix a line $l$ and a point $x$ on $l$. For any $r>0$, we let $\lambda(r)=\mathcal{H}^{1}(\partial B(x, r) \cap E)$. On $\partial B(x, r)$ we take two opposite arcs, each of length $\lambda(r) / 2$, so that $l$ passes through the midpoints of both arcs. The set obtained as the collection of all such arcs, when $r$ varies in $(0, \infty)$, is called the Bonnesen annular 
symmetrized set of $E$ and will be denoted by $E^{\text {as }}$. It is an elementary property of annular symmetrization that, for all $r>0$,

$$
|E \cap B(x, r)|=\left|E^{\mathrm{as}} \cap B(x, r)\right|,
$$

which in particular implies $|E|=\left|E^{\text {as }}\right|$. Another relevant property is

Theorem 3.4 (Bonnesen, 1924). Let $E$ be a convex set and let $r \leq R$ be, respectively, the inner and outer radii of the annulus $C_{r, R}(x)$ centered at $x$, containing $\partial E$, and having minimal width $R-r$. If $E^{\text {as }}$ is the annular symmetrization of $E$ centered at $x$ with respect to some line through $x$, then $P\left(E^{\mathrm{as}}\right) \leq P(E)$, with equality if and only if $E^{\mathrm{as}}=E$.

The proof of this theorem is not completely elementary, as one must show that if $x, r$ and $R$ are the parameters defining the optimal annulus $C_{r, R}(x)$, then both $\partial B(x, r)$ and $\partial B(x, R)$ intersect $\partial E$ in at least two distinct points (this property is crucial to show that the perimeter does not increase after the symmetrization). Moreover, this symmetrization does not preserve convexity in general (see [7]).

However, we shall not apply Bonnesen's theorem but prove instead a much more elementary property of annular symmetrization restricted to a special class of sets, on which it preserves area, smoothness and also convexity, while not increasing perimeter. To this end, for any integer $h \geq 2$ we say that a set $E \subset \mathcal{S}^{2}$ belongs to the class $\mathcal{P}(h)$ if

- $|E|=|B|$;

- $\partial E$ is of class $C^{1}$;

- there exist constants $0<\kappa_{2}<1<\kappa_{1}$ such that $\partial E \backslash B$ is a union of $h$ congruent arcs of circle with curvature $\kappa_{1}$, and similarly $\partial E \cap B$ is a union of $h$ congruent arcs of circle with curvature $\kappa_{2}$.

Note that the elements of the class $\mathcal{P}(2)$ are the ovals. Some sets belonging to $\mathcal{P}(h)$ for $h=2,3,4$ are shown in Figure 3 .
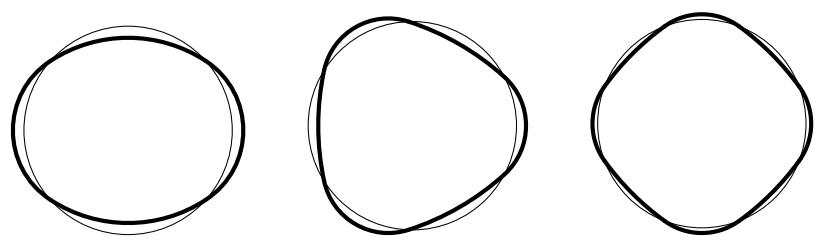

Fig. 3. Three examples of sets belonging to $\mathcal{P}(2), \mathcal{P}(3)$ and $\mathcal{P}(4)$ (from left to right). The one on the left is an oval.

Before proceeding to the proof of Theorem 3.2, we prove a lemma on the uniqueness of the optimal center for a strictly convex set, valid for any dimension $n$. We recall that $x \in \mathbb{R}^{n}$ is an optimal center for $E \in \mathcal{S}^{n}$ if $\left|B_{E}\right| \alpha(E)=\left|E \triangle\left(x+B_{E}\right)\right|$, and that the set of all optimal centers for $E$ is denoted by $\mathcal{Z}(E)$. 
Lemma 3.5. Let $E$ be a strictly convex set in $\mathbb{R}^{n}$. Then the optimal center of $E$ is unique.

Proof. Without loss of generality, we assume $B_{E}=B$. For a contradiction, suppose $x_{1}, x_{2} \in \mathcal{Z}(E)$ with $x_{1} \neq x_{2}$. By the very definition of Fraenkel asymmetry we have, for $i \in\{1,2\}$,

$$
\left|E \cap\left(x_{i}+B\right)\right|=|B|(1-\alpha(E) / 2) .
$$

We now set $x_{\lambda}=\lambda x_{1}+(1-\lambda) x_{2}$ for $\lambda \in[0,1]$, and we observe that

$$
E \cap\left(x_{\lambda}+B\right) \supseteq \lambda\left(E \cap\left(x_{1}+B\right)\right)+(1-\lambda)\left(E \cap\left(x_{2}+B\right)\right) .
$$

Since $E \cap\left(x_{\lambda}+B\right)$ is a convex set, the Brunn-Minkowski inequality (see for example [6]) together with (3.2) yields

$$
\begin{aligned}
\left|E \cap\left(x_{\lambda}+B\right)\right|^{1 / n} & \geq\left|\lambda\left(E \cap\left(x_{1}+B\right)\right)+(1-\lambda)\left(E \cap\left(x_{2}+B\right)\right)\right|^{1 / n} \\
& \geq \lambda\left|E \cap\left(x_{1}+B\right)\right|^{1 / n}+(1-\lambda)\left|E \cap\left(x_{2}+B\right)\right|^{1 / n} \\
& =|B|^{1 / n}(1-\alpha(E) / 2)^{1 / n} .
\end{aligned}
$$

This shows that $\left|E \cap\left(x_{\lambda}+B\right)\right| \geq|B|(1-\alpha(E) / 2)$. By the definition of Fraenkel asymmetry, the opposite inequality holds true as well. Thus $\left|E \cap\left(x_{\lambda}+B\right)\right|=|B|(1-\alpha(E) / 2)$. This yields equality in the Brunn-Minkowski inequality, which is equivalent to saying that, up to translation, the sets $E \cap\left(x_{\lambda}+B\right)$ are homothetic to $E \cap\left(x_{1}+B\right)$ for all $\lambda \in[0,1]$. Since they all have the same measure, they actually coincide up to translation. As a result, $\partial E \cap\left(x_{\lambda}+B\right)$ is flat in the direction of the vector $x_{2}-x_{1}$, contradicting the strict convexity of $E$.

Proof of Theorem 3.2. We recall that the first optimal coefficient is known to be $c_{1}=0$. Assuming by induction that the coefficients $c_{k}=Q^{(k)}(B)$ are finite for all $k=1, \ldots$, $m-1$, we find that $Q^{(m)}(B)=\lim _{j} E_{j}^{(m)}$, with $E_{j}^{(m)}$ satisfying the conclusion of Theorem 5.1.

Then, again by Theorem 5.1 we may suppose that, for $j$ sufficiently large, $\kappa_{j}^{(m)}=$ $H_{\partial E_{j}^{(m)}}(x)>1 / 2$ for $\mathcal{H}^{1}$-a.e. $x \in \partial E_{j}^{(m)}$, hence $E_{j}^{(m)}$ is a strictly convex set in $\mathbb{R}^{2}$. Therefore, by Lemma 3.5 we have $\mathcal{Z}\left(E_{j}^{(m)}\right)=\left\{x_{0}\right\}$ and, up to translation, we may assume that $x_{0}=0$. Hence, $B$ and $\mathbb{R}^{2} \backslash B$ are free regions for $E_{j}^{(m)}$ in the sense of Remark 4.6 below. By Theorem 5.1 and Remark 4.6 we finally deduce that $E_{j}^{(m)} \in \mathcal{P}(h)$ for some $h \geq 2$.

Now, by exploiting annular symmetrization, we will show that necessarily $E_{j}^{(m)}$ is an oval, that is, $E_{j}^{(m)} \in \mathcal{P}(2)$. Indeed, assume for contradiction that $E_{j}^{(m)} \in \mathcal{P}(h)$ for some $h>2$. To simplify notation, we drop some indices and let $E=E_{j}^{(m)}$. We will prove that the annular symmetrized set $E^{\text {as }}$ with respect to the origin of the reference system satisfies

$$
\left|E^{\mathrm{as}}\right|=|E|, \quad \alpha\left(E^{\mathrm{as}}\right)=\alpha(E), \quad P\left(E^{\mathrm{as}}\right)<P(E),
$$

contrary to the minimality of $E$. To this end, let $\rho_{1}, \rho_{2}>0$ be such that $B\left(0, \rho_{1}\right) \subset E \subset$ $B\left(0, \rho_{2}\right)$ and that both $\partial B\left(0, \rho_{1}\right)$ and $\partial B\left(0, \rho_{2}\right)$ are tangent to $\partial E$. In other words, the 
annulus $C_{\rho_{1}, \rho_{2}}=B\left(0, \rho_{2}\right) \backslash B\left(0, \rho_{1}\right)$ is of minimal thickness among those containing $\partial E$. Let $S$ be the circular sector given in polar coordinates $(r, \theta)$ by $S=[0, \infty) \times[0, \pi / h]$. Given now $\rho \in\left(\rho_{1}, \rho_{2}\right)$ we let $A_{h}(\rho)$ be the unique point of intersection of $\partial E$ with $\partial B(0, \rho)$ within the sector $S$, so $\left\{A_{h}(\rho)\right\}=\partial E \cap \partial B(0, \rho) \cap S$. We now introduce the angle $\theta_{h}(\rho) \in[0, \pi / h]$ such that, in polar coordinates, we can represent $A_{h}(\rho)=$ $\left(\rho \cos \theta_{h}(\rho), \rho \sin \theta_{h}(\rho)\right)$ and

$$
\partial E \cap S=\bigcup_{\rho \in\left[\rho_{1}, \rho_{2}\right]} A_{h}(\rho)
$$

We now have

$$
\mathcal{H}^{1}(E \cap \partial B(0, \rho))=2 h \rho \theta_{h}(\rho) .
$$

By definition of $E^{\text {as }}$ we have, for all $\rho \in\left[\rho_{1}, \rho_{2}\right]$,

$$
\mathcal{H}^{1}(E \cap \partial B(0, \rho))=\mathcal{H}^{1}\left(E^{\text {as }} \cap \partial B(0, \rho)\right)
$$

and moreover

$$
\left|E^{\text {as }} \cap B\right|=|E \cap B|, \quad\left|E^{\text {as }} \cap\left(\mathbb{R}^{2} \backslash B\right)\right|=\left|E \cap\left(\mathbb{R}^{2} \backslash B\right)\right| .
$$

By exploiting the same polar parameterization as before, we may write

$$
\partial E^{\mathrm{as}}=\bigcup_{\rho \in\left[\rho_{1}, \rho_{2}\right]} A_{2}(\rho)
$$

where $A_{2}(\rho)=\left(\rho \cos \theta_{2}(\rho), \rho \sin \theta_{2}(\rho)\right)$ for $\rho \in\left[\rho_{1}, \rho_{2}\right]$ and $\theta_{2}(\rho)$ is such that

$$
\mathcal{H}^{1}\left(E^{\text {as }} \cap \partial B(0, \rho)\right)=4 \rho \theta_{2}(\rho) .
$$

Comparing (3.3) and (3.5) we obtain

$$
\theta_{2}(\rho)=\frac{h}{2} \theta_{h}(\rho)
$$

We finally have

$$
\begin{aligned}
P\left(E^{\mathrm{as}}\right) & =4 \int_{\rho_{1}}^{\rho_{2}} \sqrt{1+\rho^{2}\left(\theta_{2}^{\prime}\right)^{2}} d \rho=4 \int_{\rho_{1}}^{\rho_{2}} \sqrt{1+\frac{h^{2}}{4} \rho^{2}\left(\theta_{h}^{\prime}\right)^{2}} d \rho \\
& <2 h \int_{\rho_{1}}^{\rho_{2}} \sqrt{1+\rho^{2}\left(\theta_{h}^{\prime}\right)^{2}} d \rho=P(E),
\end{aligned}
$$

where the last inequality follows since $h \geq 3$. To conclude the proof we show that the annular symmetrization preserves the strict convexity of our sets, i.e., the curvature $\kappa_{\mathrm{as}}(x)=H_{\partial E^{\text {as }}}(x)$ satisfies

$$
\kappa_{\mathrm{as}}(x)>0 \quad \text { for } \mathcal{H}^{1} \text {-a.e. } x \in \partial E^{\mathrm{as}} .
$$


In fact, once we have this, arguing as in Step 1 we immediately get $\mathcal{Z}\left(E^{\text {as }}\right)=\left\{x_{0}\right\}$. Then, by the symmetry of $E^{\text {as }}, x_{0}=0$, and finally, thanks to (3.4), we conclude that $\alpha\left(E^{\text {as }}\right)=\alpha(E)$.

To prove (3.8) we observe that in $B$ and $\mathbb{R}^{2} \backslash \bar{B}$ the curvature $\kappa$ of $\partial E$ can be computed through the parameterization $\theta_{h}=\theta_{h}(\rho)$ (with a slight abuse of notation, we understand $\kappa$ as a function of $\rho$ ) described before. More precisely, the well-known formula

$$
0<\kappa(\rho)=-\frac{\rho^{2}\left(\theta_{h}^{\prime}\right)^{3}+\rho \theta_{h}^{\prime \prime}+2 \theta_{h}^{\prime}}{\left(1+\left(\rho \theta_{h}^{\prime}\right)^{2}\right)^{3 / 2}}
$$

implies that $\rho^{2}\left(\theta_{h}^{\prime}\right)^{3}+\rho \theta_{h}^{\prime \prime}+2 \theta_{h}^{\prime}<0$. This, together with (3.6) and the fact that, by construction, $\theta_{h}^{\prime}(\rho)<0$, gives

$$
\kappa_{\mathrm{as}}(\rho)=-\frac{h}{2} \cdot \frac{\frac{h^{2}}{4} \rho^{2}\left(\theta_{2}^{\prime}\right)^{3}+\rho \theta_{2}^{\prime \prime}+2 \theta_{2}^{\prime}}{\left(1+\left(\frac{h}{2} \rho \theta_{2}^{\prime}\right)^{2}\right)^{3 / 2}} \geq-\frac{h}{2} \cdot \frac{\rho^{2}\left(\theta_{h}^{\prime}\right)^{3}+\rho \theta_{h}^{\prime \prime}+2 \theta_{h}^{\prime}}{\left(1+\left(\frac{h}{2} \rho \theta_{2}^{\prime}\right)^{2}\right)^{3 / 2}}>0 .
$$

Thanks to (3.7) and by the definition of $Q_{j}^{(m)}$ in (2.4), we obtain

$$
Q_{j}^{(m)}(E)>Q_{j}^{(m)}\left(E^{\mathrm{as}}\right)
$$

contrary to the minimality of $E=E_{j}^{(m)}$. This concludes the proof of the theorem.

\section{A general class of functionals}

In this section we show existence and regularity properties of minimizers for a general class of functionals defined on sets $E \in \mathcal{S}^{n}$.

Let $f, g:[0,2] \rightarrow \mathbb{R}$ be two Lipschitz-continuous functions with $g(t)$ nonnegative and zero if and only if $t=0$. We define the functional $\mathcal{F}_{f, g}: \mathcal{S}^{n} \rightarrow[-\infty, \infty]$ as follows:

$$
\mathcal{F}_{f, g}(E)= \begin{cases}\frac{\delta P(E)+f(\alpha(E))}{g(\alpha(E))} & \text { if } \alpha(E)>0 \\ \inf \left\{\liminf _{h} \mathcal{F}_{f, g}\left(E_{h}\right): \alpha\left(E_{h}\right)>0,\left|E_{h} \Delta B\right| \rightarrow 0\right\} & \text { otherwise. }\end{cases}
$$

Clearly, $\mathcal{F}_{f, g}(E)$ is invariant under isometries and dilations. In what follows, we will drop the subscripts $f$ and $g$ and simply write $\mathcal{F}$ instead of $\mathcal{F}_{f, g}$.

Given $0<\alpha_{0}<1$ we recall that

$$
\mathcal{S}_{\alpha_{0}}^{n}:=\left\{E \subset \mathcal{S}^{n}:|E|=|B|, \alpha(E) \geq \alpha_{0}\right\}
$$

and, restricting $\mathcal{F}$ to $\mathcal{S}_{\alpha_{0}}^{n}$, we state the following theorem:

Theorem 4.1 (Existence of minimizers). There exists $\hat{E} \in \mathcal{S}_{\alpha_{0}}^{n}$ such that $\mathcal{F}(\hat{E}) \leq \mathcal{F}(E)$ for all $E \in \mathcal{S}_{\alpha_{0}}^{n}$. 
Proof. We first observe that the subclass $\mathcal{S}_{\alpha_{0}}^{n}$ is closed with respect to the $L^{1}$-convergence of sets. We now fix a minimizing sequence $\left(E_{h}\right)_{h} \subset \mathcal{S}_{\alpha_{0}}^{n}$ for $\mathcal{F}$ and assume $\left|E_{h}\right|=|B|$ for all $h$. We can of course assume that $\mathcal{F}\left(E_{h}\right) \leq C<\infty$ for all $h$ and for some constant $C>0$. Therefore,

$$
P\left(E_{h}\right) \leq P(B)+C g\left(\alpha\left(E_{h}\right)\right)-f(\alpha(E)) \leq P(B)+C \max g-\min f<\infty
$$

for all $h$. As a consequence, by a well-known compactness result for families of sets with equibounded perimeter (see for instance [21]), $\left(E_{h}\right)_{h}$ is sequentially relatively compact in $L_{\text {loc }}^{1}\left(\mathbb{R}^{n}\right)$. Starting from $E_{h}$ we can construct a new sequence $\hat{E}_{h}$ that is a uniformly bounded minimizing sequence for $\mathcal{F}$ converging to a limit set $\hat{E}$. To this end, we shall adapt to our case an argument originally employed by Almgren [1].

First, by a standard concentration-compactness argument, one can prove that there exists $\beta_{0}>0$ (depending only on the data of the problem, and not on the sequence $\left.\left(E_{h}\right)_{h}\right)$ and $\left\{x_{h}^{0}\right\}_{h} \subset \mathbb{R}^{n}$ such that

$$
\left|E_{h} \cap\left(x_{h}^{0}+B\right)\right| \geq \beta_{0} .
$$

Of course, we can assume that $x_{h}^{0}$ is an optimal center for $E_{h}$, that is, $\alpha\left(E_{h}\right)=$ $\left|E_{h} \triangle\left(x_{h}^{0}+B\right)\right| /|B|$. The functional $\mathcal{F}$ is invariant with respect to translations, thus the translated sequence $E_{h}^{0}:=E_{h}-x_{h}^{0}$ is still minimizing and, at the same time, satisfies $\left|E_{h}^{0} \cap B\right| \geq \beta_{0}$. Up to subsequences, we can assume that $E_{h}^{0}$ converges to $E^{0}$ in the $L_{\text {loc }}^{1}$-topology.

Now, we face two possibilities: either $\left|E^{0}\right|=|B|$ and this would immediately imply that $E_{h}^{0} \rightarrow E^{0}$ in $L^{1}$ (in this case we are done), or $\beta_{0} \leq\left|E^{0}\right|<|B|$. The latter possibility corresponds to a "loss of mass at infinity". In order to deal with this case, we first study the minimality of $E^{0}$ with respect to perimeter. By the argument in the proof of [12, Lemma 3.3(ii)], $P\left(E^{0}\right) \leq P(F)$ for all measurable $F \subset \mathbb{R}^{n}$ such that $|F|=\left|E^{0}\right|$ and $F \triangle E^{0}$ is compactly contained in $\mathbb{R}^{n} \backslash B(0, R)$ for a sufficienty large $R$. As a consequence, by well-known results on minimizers of perimeter subject to a volume constraint, we infer that $E^{0}$ is necessarily bounded. Choose $R_{0}>0$ such that $E_{0} \subset B\left(0, R_{0}\right)$. Since $B$ is an optimal ball for $E_{h}^{0}$, and since $E_{h}^{0}$ converges to $E^{0}$ in $L_{\text {loc }}^{1}$, we set

$$
\gamma:=2\left|B \backslash E^{0}\right| /|B|=\lim _{h} \alpha\left(E_{h}^{0}\right) .
$$

Clearly, $\alpha_{0} \leq \gamma<2$. In the case $\gamma<1$, the set $\tilde{E}=E^{0} \cup B_{0}$ minimizes the functional $\mathcal{F}$, where $B_{0}$ is a ball such that $B_{0} \cap B\left(0, R_{0}+2\right)=\emptyset$ and $\left|E^{0} \cup B_{0}\right|=\left|E^{0}\right|+\left|B_{0}\right|=|B|$. Indeed, $\alpha(\tilde{E})=\gamma$ and $P(\tilde{E}) \leq \liminf _{h} P\left(E_{h}^{0}\right)$. In the case $\gamma \geq 1$ we proceed differently. Since we are facing a loss of mass in the limit, $\left|E_{h}^{0} \backslash B\left(0, R_{0}\right)\right| \rightarrow|B|-\left|E^{0}\right|>0$ as $h \rightarrow \infty$. Hence we can find $\beta_{1}>0$ and $\left\{x_{h}^{1}\right\}_{h} \subset \mathbb{R}^{n}$ such that, as before, $\left|E_{h}^{0} \cap\left(x_{h}^{1}+B\right)\right|$ $\geq \beta_{1}$. Note that $\left|x_{h}^{1}\right| \rightarrow \infty$ as $h \rightarrow \infty$, otherwise by compactness we would contradict the inclusion $E^{0} \subset B\left(0, R_{0}\right)$. We may also assume that

$$
x_{h}^{1} \in \underset{x \in \mathbb{R}^{n} \backslash B\left(0, R_{0}+2\right)}{\arg \max }\left|E_{h}^{0} \cap(x+B)\right| .
$$


Arguing as before, we can extract a subsequence of $E_{h}^{0}$ (that we do not relabel) such that $E_{h}^{1}:=E_{h}^{0}-x_{h}^{1}$ converges to $E^{1}$ in $L_{\text {loc }}^{1}$ as $h \rightarrow \infty$. Moreover, we let $R_{1}>0$ be such that $E^{1} \subset B\left(0, R_{1}\right)$.

Now, we show that there exists a constant $C>1$ depending only on the data of the problem (and not on the minimizing sequence $E_{h}$ ) such that

$$
\frac{1}{C}\left|E^{0}\right| \leq\left|E^{1}\right| \leq C\left|E^{0}\right|
$$

To prove (4.2) it is enough to show the first inequality, i.e. $\left|E^{0}\right| \leq C\left|E^{1}\right|$ for some uniform $C>1$ (the other is implied by the estimate $\left|E^{0}\right| \geq \beta_{0}$ shown above). Indeed, assume $\left|E^{1}\right| \leq\left|E^{0}\right|$ (otherwise there is nothing to prove). We consider the following modified sequence:

$$
\tilde{E}_{h}=\left(\lambda_{h} E^{0}\right) \cup\left[E_{h}^{0} \backslash\left(B\left(0,2 R_{0}\right) \cup B\left(x_{h}^{1}, R_{1}\right)\right)\right],
$$

where $\lambda_{h}>1$ is such that $\left|\tilde{E}_{h}\right|=\left|E_{h}\right|=|B|$. Then $\lambda_{h} \rightarrow\left(1+\left|E^{1}\right| /\left|E^{0}\right|\right)^{1 / n}$ as $h \rightarrow \infty$, and by Bernoulli's inequality we also have

$$
1 \leq \lambda_{h} \leq 1+\frac{\left|E^{1}\right|}{n\left|E^{0}\right|}+\varepsilon_{h},
$$

where $\varepsilon_{h} \rightarrow 0$ as $h \rightarrow \infty$. Since $\left|E^{1}\right|<\left|E^{0}\right|$, we can assume without loss of generality that $1 \leq \lambda_{h}<2$ for all $h$. We now set $\alpha_{h}=\alpha\left(E_{h}\right), \tilde{\alpha}_{h}=\alpha\left(\tilde{E}_{h}\right)$, and $\delta_{h}=\left|\tilde{\alpha}_{h}-\alpha_{h}\right|$. In what follows, to simplify notation and not to overburden the reader, we let "n.t." stand for $O\left(\varepsilon_{h}\right)+o\left(\left|E^{1}\right| /\left|E^{0}\right|\right)$. We first show that

$$
\delta_{h} \leq 2 \frac{\left|E^{1}\right|}{\left|E^{0}\right|}+\text { n.t. }
$$

Indeed, we recall that here

$$
\alpha_{h}=2\left|B \backslash E_{h}^{0}\right| /|B| \rightarrow \gamma \geq 1
$$

as $h \rightarrow \infty$. Then, since $\lambda_{h} \geq 1$ we get

$$
\begin{aligned}
\alpha_{h} & =\frac{2\left|B \backslash E^{0}\right|}{|B|}+\text { n.t. }=\frac{2 \lambda_{h}^{-n}}{|B|}\left|\lambda_{h} B \backslash \lambda_{h} E^{0}\right|+\text { n.t. } \geq \frac{2 \lambda_{h}^{-n}}{|B|}\left|B \backslash \lambda_{h} E^{0}\right|+\text { n.t. } \\
& \geq \lambda_{h}^{-n} \tilde{\alpha}_{h}+\text { n.t. },
\end{aligned}
$$

whence

$$
\tilde{\alpha}_{h} \leq \lambda_{h}^{n} \alpha_{h}+\text { n.t. } \leq \alpha_{h}+\frac{\left|E^{1}\right|}{\left|E^{0}\right|} \alpha_{h}+\text { n.t. } \leq \alpha_{h}+2 \frac{\left|E^{1}\right|}{\left|E^{0}\right|}+\text { n.t. }
$$

We now consider the following two cases.

Case 1: there exists an optimal ball $\tilde{B}_{h}$ for $\tilde{E}_{h}$ such that $\tilde{B}_{h} \cap B\left(0,2 R_{0}\right)=\emptyset$. In this case

$$
\tilde{\alpha}_{h}=2\left|\tilde{B}_{h} \backslash \tilde{E}_{h}\right| /|B| \geq 2\left|\tilde{B}_{h} \backslash E_{h}^{0}\right| /|B| \geq \alpha_{h} .
$$


Case 2: any optimal ball $\tilde{B}_{h}$ for $\tilde{E}_{h}$ satisfies $\tilde{B}_{h} \cap B\left(0,2 R_{0}\right) \neq \emptyset$. In this case, if we set $\tilde{B}_{h}=B\left(\tilde{x}_{h}, 1\right)$ and recall that $B$ is an optimal ball for $E_{h}$, for all $h$, we obtain

$$
\begin{aligned}
\tilde{\alpha}_{h} & =2 \frac{\left|\tilde{B}_{h} \backslash \lambda_{h} E^{0}\right|}{|B|}+\text { n.t. }=2 \frac{\lambda_{h}^{n}}{|B|}\left|\frac{\tilde{B}_{h}}{\lambda_{h}} \backslash E^{0}\right|+\text { n.t. } \\
& \geq 2 \frac{\lambda_{h}^{n}}{|B|}\left[\left|B\left(\tilde{x}_{h} / \lambda_{h}, 1\right) \backslash E^{0}\right|-|B|\left(1-\lambda_{h}^{-n}\right)\right]+\text { n.t. } \\
& \geq \lambda_{h}^{n} \alpha_{h}-2\left(\lambda_{h}^{n}-1\right)+\text { n.t. }=\alpha_{h}+\left(\alpha_{h}-2\right)\left(\lambda_{h}^{n}-1\right)+\text { n.t. } \\
& \geq \alpha_{h}-2 \frac{\left|E^{1}\right|}{\left|E^{0}\right|}+\text { n.t. }
\end{aligned}
$$

which combined with (4.7) and (4.6) gives (4.4).

Assume for contradiction that the ratio $\left|E^{1}\right| /\left|E^{0}\right|$ is not bounded below by a positive constant that depends only on the data of the problem. Then by (4.4) and (4.5) we have $\tilde{\alpha}_{h} \geq \alpha_{0}$. Therefore, $\tilde{E}_{h} \in \mathcal{S}_{\alpha_{0}}^{n}$, so that we are allowed to compare $\mathcal{F}\left(\tilde{E}_{h}\right)$ with $\mathcal{F}\left(E_{h}^{0}\right)$. Thanks to the hypothesis on $g$, we also have, for $h$ large enough, $g\left(\alpha_{h}\right)-\operatorname{Lip}(g) \delta_{h}>0$. Thus by (4.4), up to a suitable choice of the radii $R_{0}$ and $R_{1}$ (for details we refer to [12, proof of Lemma 3.3(ii)]), we obtain

$$
\begin{aligned}
\mathcal{F}\left(\tilde{E}_{h}\right)= & \frac{\delta P\left(\tilde{E}_{h}\right)+f\left(\tilde{\alpha}_{h}\right)}{g\left(\tilde{\alpha}_{h}\right)} \\
\leq & \frac{P\left(\lambda_{h} E^{0}\right)+P\left(\tilde{E}_{h} \backslash B\left(0,2 R_{0}\right)\right)-P(B)+P(B)\left[f\left(\alpha_{h}\right)+\operatorname{Lip}(f) \delta_{h}\right]}{P(B)\left[g\left(\alpha_{h}\right)-\operatorname{Lip}(g) \delta_{h}\right]} \\
\leq & \frac{\left(\lambda_{h}^{n-1}-1\right) P\left(E^{0}\right)+P\left(E_{h}^{0}\right)-P(B)-P\left(E_{h}^{0} \cap B\left(x_{h}^{1}, R_{1}\right)\right)}{P(B)\left[g\left(\alpha_{h}\right)-\operatorname{Lip}(g) \delta_{h}\right]} \\
& +\frac{f\left(\alpha_{h}\right)+\operatorname{Lip}(f) \delta_{h}}{g\left(\alpha_{h}\right)-\operatorname{Lip}(g) \delta_{h}}+o(1) .
\end{aligned}
$$

Since

$$
\liminf _{h} P\left(E_{h}^{0} \cap B\left(x_{h}^{1}, R_{1}\right)\right) \geq P\left(E^{1}\right),
$$

by exploiting the isoperimetric inequality we get

$$
\begin{aligned}
\mathcal{F}\left(\tilde{E}_{h}\right) \leq & \frac{\left(\lambda_{h}^{n-1}-1\right) P\left(E^{0}\right)+P\left(E_{h}^{0}\right)-P(B)-n \omega_{n}^{1 / n}\left|E^{1}\right|^{n-1 / n}}{P(B)\left[g\left(\alpha_{h}\right)-\operatorname{Lip}(g) \delta_{h}\right]} \\
& +\frac{f\left(\alpha_{h}\right)+\operatorname{Lip}(f) \delta_{h}}{g\left(\alpha_{h}\right)-\operatorname{Lip}(g) \delta_{h}}+o(1) .
\end{aligned}
$$

Combining (4.3) and (4.9), after some straightforward computations we get

$$
\mathcal{F}\left(\tilde{E}_{h}\right) \leq \mathcal{F}\left(E_{h}^{0}\right)-\frac{\omega_{n}^{1 / n-1}}{\max g}\left|E^{1}\right|^{(n-1) / n}+o(1),
$$

which contradicts the optimality of the sequence $E_{h}^{0}$, thus proving (4.2). 
Since the volume of $E_{h}$ equals $|B|$, an immediate consequence of (4.2) is that there exists a finite family $\left\{E^{0}, E^{1}, \ldots, E^{N}\right\}$ of sets of finite perimeter, obtained as $L_{\text {loc }}^{1}$-limits of suitably translated subsequences of the initial minimizing sequence $E_{h}$, which satisfy

(a) $\left|E^{0}\right|+\cdots+\left|E^{N}\right|=|B|$;

(b) $\liminf _{h} P\left(E_{h}\right) \geq \sum_{i=0}^{N} P\left(E^{i}\right)$;

(c) $\alpha\left(E_{h}\right) \rightarrow \min _{i=0, \ldots, N} \min _{x \in \mathbb{R}^{n}} 2\left|(x+B) \backslash E^{i}\right| /|B|$ as $h \rightarrow \infty$.

The proof of (a) and (b) is routine. On the other hand, (c) follows from the fact that given $i, j \in\{1, \ldots, N\}$ with $i \neq j$, the two sets $E^{i}$ and $E^{j}$ are obtained as limits in $L_{\text {loc }}^{1}$ of a subsequence of $\left(E_{h}\right)_{h}$, with suitable translation vectors $x_{h}^{i}$ and $x_{h}^{j}$ respectively that satisfy $\lim _{h}\left|x_{h}^{i}-x_{h}^{j}\right|=\infty$.

We can now construct a minimizer of $\mathcal{F}$ by simply setting

$$
\hat{E}=E^{0} \cup\left(v+E^{1}\right) \cup\left(2 v+E^{2}\right) \cup \cdots \cup\left(N v+E^{n}\right),
$$

where $v \in \mathbb{R}^{n}$ is any vector such that $E^{i} \subset B(0,|v| / 2-2)$ for all $i=0, \ldots, N$. In this way, we guarantee by (a) above that $|\hat{E}|=|B|$ and, by (c), that

$$
\alpha(\hat{E})=\min _{i=0, \ldots, N} \min _{x \in \mathbb{R}^{n}} \frac{\left|(x+B) \backslash E^{i}\right|}{|B|}=\lim _{h} \alpha\left(E_{h}\right) .
$$

Finally, by (b) and (4.10) we conclude that $\hat{E}$ is a minimizer of $\mathcal{F}$.

In the lemma below we recall an elementary but useful estimate of a difference of asymmetries in terms of the volume of the symmetric difference of the corresponding sets.

Lemma 4.2. Let $E \in \mathcal{S}^{n}$ with $|E|=|B|=\omega_{n}$. For all $x \in \mathbb{R}^{n}$ and for any $F \in \mathcal{S}^{n}$ with $E \triangle F \subset \subset B(x, 1 / 2)$,

$$
|\alpha(E)-\alpha(F)| \leq \frac{2^{n+2}}{\left(2^{n}-1\right) \omega_{n}}|E \Delta F| .
$$

The next, crucial theorem asserts the $\Lambda$-minimality of the minimizers of the functional (4.1).

Theorem 4.3 ( $\Lambda$-minimality). Let $\mathcal{F}$ be the functional defined in (4.1). Then there exists $\Lambda>0$ such that any minimizer $E \in \mathcal{S}^{n}$ of $\mathcal{F}$ with $|E|=|B|$ is a $\Lambda$-minimizer of the perimeter.

Proof. Of course, if $\alpha(E)=0$ there is nothing to prove, since $E$ is a ball (and thus a wellknown $\Lambda$-minimizer of the perimeter) up to null sets. We now assume $\alpha(E)>0$ and fix $x \in \mathbb{R}^{n}$ and a compact variation $F$ of $E$ in $B(x, 1 / 2)$. It is not restrictive to assume that $P(F) \leq P(E)$ and $\alpha(F)>0$. Since $\mathcal{F}(E) \leq \mathcal{F}(F)$, we have

$$
(\delta P(E)+f(\alpha(E))) g(\alpha(F)) \leq g(\alpha(E))(\delta P(F)+f(\alpha(F))) .
$$

Then, combining Lemma 4.2 and the Lipschitz continuity of $g$, we have

$$
|g(\alpha(E))-g(\alpha(F))| \leq \operatorname{Lip}(g)|\alpha(E)-\alpha(F)| \leq C_{n, g}|E \triangle F|
$$


with $C_{n, g}=\operatorname{Lip}(g) \frac{2^{n+2}}{\left(2^{n}-1\right) \omega_{n}}$. We now set

$$
\chi=\operatorname{sgn}(\delta P(E)+f(\alpha(E)))=\operatorname{sgn} \mathcal{F}(E)
$$

and observe that by (4.12),

$$
(\delta P(E)+f(\alpha(E)))\left(g(\alpha(E))-\chi C_{n, g}|E \triangle F|\right) \leq(\delta P(E)+f(\alpha(E))) g(\alpha(F)),
$$

thus plugging (4.13) into (4.11) and dividing by $g(\alpha(E))$ we get

$$
\delta P(E)-\delta P(F) \leq f(\alpha(F))-f(\alpha(E))+C_{n, g}|\mathcal{F}(E)| \cdot|E \triangle F| .
$$

On recalling that $f$ is Lipschitz, we have (4.12) with $f$ replacing $g$, and therefore

$$
\delta P(E)-\delta P(F) \leq\left(C_{n, f}+C_{n, g}|\mathcal{F}(E)|\right)|E \triangle F| .
$$

Then, we note that

$$
\delta P(E)-\delta P(F)=\frac{P(E)-\frac{P(B)}{P\left(B_{F}\right)} P(F)}{P(B)}
$$

and

$$
\begin{aligned}
\frac{P(B)}{P\left(B_{F}\right)} & =\left(\frac{|E|}{|F|}\right)^{(n-1) / n}=\left(1+\frac{|E|-|F|}{|F|}\right)^{(n-1) / n} \leq\left(1+\frac{|E \Delta F|}{|F|}\right)^{(n-1) / n} \\
& \leq 1+\frac{n-1}{n} \cdot \frac{|E \Delta F|}{|F|} \leq 1+\frac{4(n-1)}{3 n|B|} \cdot|E \Delta F|
\end{aligned}
$$

where we have used the Bernoulli inequality and the fact that $E \triangle F \subset \subset B(x, 1 / 2)$ implies $|F| \geq|B|-|B(x, 1 / 2)|=\frac{3}{4}|B|$. Finally, using (4.16) and (4.17) we can rewrite (4.15) as

$$
\begin{aligned}
P(E) & \leq P(F)+|E \Delta F|\left(C_{n, f}+C_{n, g}|\mathcal{F}(E)| P(B)+\frac{4(n-1)}{3 n|B|} P(E)\right) \\
& =P(F)+\Lambda|E \triangle F|
\end{aligned}
$$

where the constant

$$
\Lambda=C_{n, f}+C_{n, g}|\mathcal{F}(E)| P(B)+\frac{4(n-1)}{3 n|B|} P(E)
$$

depends only on the dimension $n$ and on the functions $f$ and $g$.

As a consequence of Theorems 4.1, 4.3 and 2.1 one obtains the following

Theorem 4.4 (Regularity). Let $\mathcal{F}$ be the functional defined in (4.1) and let $E \in \mathcal{S}^{n}$ be a minimizer of $\mathcal{F}$ with $|E|=|B|$. Then $\partial^{*} E$ is of class $C^{1, \eta}$ for any $\eta \in(0,1)\left(C^{1,1}\right.$ for $n=2$ ), while the singular set $\partial E \backslash \partial^{*} E$ has Hausdorff dimension $\leq n-8$. 
In the following lemma, we let $E$ be a minimizer of $\mathcal{F}$ and we show that the (scalar) weak mean curvature $H$ of $\partial E$ belongs to $L^{\infty}(\partial E)$. Moreover, we compute a first variation inequality of $\mathcal{F}$ at $E$ that translates into a quantitative estimate of the oscillation of the mean curvature.

Lemma 4.5. Let $E$ be a minimizer of $\mathcal{F}$. Then $\partial^{*} E$ has the weak mean curvature $H \in$ $L^{\infty}\left(\partial^{*} E\right)$ (with orientation induced by the inner normal to E). Moreover, for $\mathcal{H}^{n-1}$-a.e. $x, y \in \partial^{*} E$, one has

$$
|H(x)-H(y)| \leq \frac{n}{n-1}(|\mathcal{F}(E)| \operatorname{Lip}(g)+\operatorname{Lip}(f)) .
$$

Proof. To prove the theorem we consider a "parametric inflation-deflation", that will lead to the first variation inequality (4.19).

Fix $x_{1}, x_{2} \in \partial^{*} E, x_{1} \neq x_{2}$. By Theorem 4.4, there exist $r>0$ such that, for $m=1,2$,

$$
\partial E \cap B\left(x_{m}, r\right)=\partial^{*} E \cap B\left(x_{m}, r\right)
$$

is the graph of a smooth function $f_{m}$ defined on an open set $A_{m} \subset \mathbb{R}^{n-1}$, with respect to a suitable reference frame, so that the set $E \cap B\left(x_{m}, r\right)$ "lies below" the graph of $f_{m}$. For $m=1,2$ we take $\varphi_{m} \in C_{c}^{1}\left(A_{m}\right)$ such that $\varphi_{m} \geq 0$ and

$$
\int_{A_{m}} \varphi_{m}=1
$$

Let $\varepsilon>0$ be such that, setting $f_{m, t}(w)=f_{m}(w)+(-1)^{m} t \varphi_{m}(w)$ for $w \in A_{m}$, one has $\operatorname{gr}\left(f_{m, t}\right) \subset B\left(x_{m}, r\right)$ for all $t \in(-\varepsilon, \varepsilon)$. We use the functions $f_{m, t}, m=1,2$, to modify the set $E$, i.e. we define $E_{t}$ so that $E_{t} \triangle E$ is compactly contained in $B\left(x_{1}, r\right) \cup B\left(x_{2}, r\right)$, with $\partial E_{t} \cap B\left(x_{m}, r\right)=\operatorname{gr}\left(f_{m, t}\right)$ for $m=1,2$. By (4.20) one immediately deduces that $\left|E_{t}\right|=|E|$. Moreover, by a standard computation one obtains

$$
\left.\frac{1}{n-1} \frac{d}{d t} P\left(E_{t}\right)\right|_{t=0}=\int_{A_{1}} h_{1} \varphi_{1}-\int_{A_{2}} h_{2} \varphi_{2},
$$

where for $m=1,2$ and in the sense of distributions

$$
h_{m}(v):=H\left(v, f_{m}(v)\right)=-\frac{1}{n-1} \operatorname{div}\left(\frac{\nabla f_{m}(v)}{\sqrt{1+\left|\nabla f_{m}(v)\right|^{2}}}\right) .
$$

Then, by Theorem 4.7.4 in [3], the $L^{\infty}$-norm of $H$ over $\partial E$ turns out to be bounded by a constant depending only on $\Lambda$ and on the dimension $n$.

By the definition of $E_{t}$ one can verify that, for $t>0$,

$$
\left|\alpha\left(E_{t}\right)-\alpha(E)\right| \leq t / \omega_{n}
$$


By (4.21) and (4.22), for $t>0$, we also have that

$$
\begin{aligned}
\mathcal{F}\left(E_{t}\right) & =\frac{P\left(E_{t}\right)-P(B)+P(B) f\left(\alpha\left(E_{t}\right)\right)}{P(B) g\left(\alpha\left(E_{t}\right)\right)} \\
& =\frac{P(E)-P(B)+P(B) f\left(\alpha\left(E_{t}\right)\right)}{P(B) g\left(\alpha\left(E_{t}\right)\right)}+\left.\frac{t}{P(B) g\left(\alpha\left(E_{t}\right)\right)} \frac{d}{d t} P\left(E_{t}\right)\right|_{t=0}+o(t) \\
& =\mathcal{F}(E) \cdot \frac{g(\alpha(E))}{g\left(\alpha\left(E_{t}\right)\right)}+\frac{f\left(\alpha\left(E_{t}\right)\right)-f(\alpha(E))}{g\left(\alpha\left(E_{t}\right)\right)}+\left.\frac{t}{n \omega_{n} g\left(\alpha\left(E_{t}\right)\right)} \frac{d}{d t} P\left(E_{t}\right)\right|_{t=0}+o(t) \\
& \leq \mathcal{F}(E)+\frac{t}{n \omega_{n} g\left(\alpha\left(E_{t}\right)\right)}\left(n|\mathcal{F}(E)| \operatorname{Lip}(g)+n \operatorname{Lip}(f)+\left.\frac{d}{d t} P\left(E_{t}\right)\right|_{t=0}\right)+o(t) .
\end{aligned}
$$

Exploiting now the minimality hypothesis $\mathcal{F}(E) \leq \mathcal{F}\left(E_{t}\right)$ in the previous inequality, dividing by $t>0$, multiplying by $n \omega_{n} g\left(\alpha\left(E_{t}\right)\right)$, and finally taking the limit as $t \rightarrow 0$, we obtain

$$
0 \leq\left.\frac{d}{d t} P\left(E_{t}\right)\right|_{t=0}+n|\mathcal{F}(E)| \operatorname{Lip}(g)+n \operatorname{Lip}(f) .
$$

Let now $w_{m} \in A_{m}$ be a Lebesgue point for $h_{f_{m}}, m=1,2$. On choosing a sequence $\left(\varphi_{m}^{k}\right)_{k} \subset C_{c}^{1}\left(A_{m}\right)$ of nonnegative mollifiers such that

$$
\lim _{k} \int_{A_{m}} h_{f_{m}} \varphi_{m}^{k}=h_{f_{m}}\left(w_{m}\right)
$$

for $m=1,2$, we find that for $E_{t}^{k}$ defined as before, but with $\varphi_{m}^{k}$ replacing $\varphi_{m}$,

$$
\begin{aligned}
\left.\frac{1}{n-1} \lim _{k} \frac{d}{d t} P\left(E_{t}^{k}\right)\right|_{t=0} & =\lim _{k} \int_{A_{1}} h_{f_{1}} \varphi_{1}^{k}-\int_{A_{2}} h_{f_{2}} \varphi_{2}^{k} \\
& =h_{f_{1}}\left(w_{1}\right)-h_{f_{2}}\left(w_{2}\right) .
\end{aligned}
$$

Moreover, from (4.23) with $E_{t}^{k}$ in place of $E_{t}$, thanks to (4.24) we get

$$
h_{f_{2}}\left(w_{2}\right)-h_{f_{1}}\left(w_{1}\right)=-\left.\frac{1}{n-1} \lim _{k} \frac{d}{d t} P\left(E_{t}^{k}\right)\right|_{t=0} \leq \frac{n}{n-1}(|\mathcal{F}(E)| \operatorname{Lip}(g)+\operatorname{Lip}(f)) \text {. }
$$

Finally, the proof of (4.19) is completed by exchanging the roles of $x_{1}$ and $x_{2}$.

Remark 4.6. Under the hypotheses of the previous theorem, if we additionally suppose that $f, g$ are $C^{1}$ functions, then arguing as above we obtain, for $\mathcal{H}^{n-1}$-a.e. $x, y \in \partial^{*} E$,

$$
|H(x)-H(y)| \leq \frac{n}{n-1}\left(|\mathcal{F}(E)| \cdot\left|g^{\prime}(\alpha(E))\right|+\left|f^{\prime}(\alpha(E))\right|\right) .
$$

Moreover, if $x, y \in \partial^{*} E$ are such that the inflation-deflation procedure in a small neighborhood of $\{x, y\}$ does not change the asymmetry (i.e., if $\alpha\left(E_{t}\right)=\alpha(E)$ for $t$ small) then we get $H(x)=H(y)$. This property is satisfied, in particular, by any pair $(x, y)$ of points belonging to the same free region (see Section 2 for the definition). Indeed, it is not difficult to show that small inflations-deflations localized in a free region $A \subset \Omega$ do not change the asymmetry, thus implying that $A \cap \partial E$ has constant mean curvature. Clearly, the value of the weak mean curvature can change from one free region to another. 


\section{The Iterative Selection Principle}

We start by stating the $n$-dimensional version of our Iterative Selection Principle. The notation used in this section has been introduced in Section 2.

Theorem 5.1 (Iterative Selection Principle). Let $m \geq 2$ and assume that $Q^{(k)}(B) \in \mathbb{R}$ for all $k=1, \ldots, m-1$. Then there exists a sequence $\left(E_{j}^{(m)}\right)_{j} \subset \mathcal{S}^{n}$ of sets such that

(i) $\left|E_{j}^{(m)}\right|=|B|, \alpha\left(E_{j}^{(m)}\right)>0$ and $\alpha\left(E_{j}^{(m)}\right) \rightarrow 0$ as $j \rightarrow \infty$;

(ii) $Q^{(m)}\left(E_{j}^{(m)}\right) \rightarrow Q^{(m)}(B)$ as $j \rightarrow \infty$;

(iii) for each $j$ there exists a function $u_{j}^{(m)} \in C^{1, \gamma}(\partial B)$, for all $0<\gamma<1$, such that

$$
\partial E_{j}^{(m)}=\left\{\left(1+u_{j}^{(m)}(x)\right) x: x \in \partial B\right\}
$$

and $u_{j}^{(m)} \rightarrow 0$ in the $C^{1}$-norm as $j \rightarrow \infty$;

(iv) $\partial E_{j}^{(m)}$ has weak mean curvature $H_{j}^{(m)} \in L^{\infty}\left(\partial E_{j}^{(m)}\right)$ and

$$
\left\|H_{j}^{(m)}-1\right\|_{L^{\infty}\left(\partial E_{j}^{(m)}\right)} \rightarrow 0 \quad \text { as } j \rightarrow \infty .
$$

Moreover, $H_{j}^{(m)}$ is constant on any free region for $E_{j}^{(m)}$.

Note that in the Iterative Selection Principle we do not assume that $Q^{(m)}(B)$ is finite. On one hand, the case $Q^{(m)}(B)=\infty$ is trivial since the conclusion of the theorem is satisfied by any sufficiently nice sequence of sets with positive asymmetry and converging to $B$ (for instance, by a sequence of ellipsoids). On the other hand, the case $Q^{(m)}(B)=-\infty$ can interestingly enough be treated the same way as the finite case.

The proof of Theorem 5.1 will require some intermediate results. Here we follow more or less the proof scheme adopted in [12]. First, we make the following observation:

Lemma 5.2 (Ball exclusion). Assume $Q^{(m)}$ is well-defined. If $\left(F_{h}\right)_{h}$ is a minimizing sequence for $Q_{j}^{(m)}$, then there exist $\beta>0$ and $h_{0} \in \mathbb{N}$ such that $\alpha\left(F_{h}\right) \geq \beta$ for all $h \geq h_{0}$ (in other words, $F_{h}$ cannot converge to the ball $B$ ).

Proof. For contradiction, assume $\alpha\left(F_{h}\right) \rightarrow 0$ as $h \rightarrow \infty$ (up to subsequences). By the very definition of $Q^{(m-1)}(B)$, thanks to its finiteness, we have

$$
Q^{(m-1)}(B) \leq Q^{(m-1)}\left(F_{h}\right)+o(1)
$$

As a consequence,

$$
\delta P\left(F_{h}\right)-\psi_{m-1}\left(\alpha\left(F_{h}\right)\right) \geq Q^{(m-1)}(B) \alpha\left(F_{h}\right)^{m-1}+o\left(\alpha\left(F_{h}\right)^{m-1}\right) .
$$

On the other hand, we have $\psi_{m}(\alpha)=\psi_{m-1}(\alpha)+Q^{(m-1)}(B) \alpha^{m-1}$, therefore thanks to (5.1), and owing to the definition of $Q_{j}^{(m)}$, we obtain

$Q_{j}^{(m)}\left(F_{h}\right) \geq \frac{\alpha\left(F_{h}\right)^{m-1}\left(\alpha\left(F_{h}\right) / \alpha_{j}^{(m)}-1\right)^{2}+o\left(\alpha\left(F_{h}\right)^{m-1}\right)}{\alpha\left(F_{h}\right)^{m}}=\frac{\alpha\left(F_{h}\right)^{m-1}+o\left(\alpha\left(F_{h}\right)^{m-1}\right)}{\alpha\left(F_{h}\right)^{m}}$. 
Since the right-hand side tends to $\infty$ as $h \rightarrow \infty$, while the functional $Q_{j}^{(m)}$ is not identically $\infty$, we get a contradiction.

On combining Theorem 4.1 with Lemma 5.2 we can prove the following

Proposition 5.3. Assume $Q^{(m)}$ is well-defined. Then the associated penalized functional $Q_{j}^{(m)}$ admits a minimizer $E_{j}^{(m)} \in \mathcal{S}^{n}$ with $\alpha\left(E_{j}^{(m)}\right)>0$.

Proof. We first observe that, on choosing $f_{j}(\alpha)=\psi_{m}(\alpha)+\alpha^{m-1}\left(\alpha / \alpha_{j}^{(m)}-1\right)^{2}$ and $g(\alpha)=\alpha^{m}$, we have $\mathcal{F}_{f_{j}, g}=Q_{j}^{(m)}$. Then the conclusion is a direct consequence of Theorem 4.1 and of Lemma 5.2.

Lemma 5.4. Assume $Q^{(m)}$ is well-defined and $Q^{(m)}(B)>-\infty$. Then there exists $\lambda_{m} \in \mathbb{R}$ such that

$$
Q^{(m)}(E) \geq \lambda_{m} \quad \text { for all } E \in \mathcal{S}^{n} .
$$

Proof. Aiming at a contradiction, if there existed a sequence $\left(F_{h}\right)_{h}$ of sets in $\mathcal{S}^{n}$ satisfying $Q^{(m)}\left(F_{h}\right) \rightarrow-\infty$ as $h \rightarrow \infty$, by the assumption on $Q^{(m)}(B)$ we would find $\beta>0$ such that $\alpha\left(F_{h}\right) \geq \beta$ for $h$ sufficiently large. Consequently, from the very definition of $Q^{(m)}$ and the fact that $\delta P\left(F_{h}\right) \geq 0$ we would deduce that

$$
Q^{(m)}\left(F_{h}\right) \geq \frac{-\sup \left\{\psi_{m}(\alpha): \beta \leq \alpha<2\right\}}{\alpha\left(F_{h}\right)} \geq-\frac{\left|\sup \left\{\psi_{m}(\alpha): \beta \leq \alpha<2\right\}\right|}{\beta},
$$

which leads to a contradiction on observing that, by the assumptions, $\sup \left\{\psi_{m}(\alpha): \beta \leq\right.$ $\alpha<2\} \in \mathbb{R}$.

The next proposition deals with the asymptotic behavior, as $j \rightarrow \infty$, of the sequences $\left(E_{j}^{(m)}\right)_{j},\left(Q^{(m)}\left(E_{j}^{(m)}\right)\right)_{j}$ and $\left(\alpha\left(E_{j}^{(m)}\right)\right)_{j}$.

Lemma 5.5. Let $Q^{(m)}$ be well-defined with $Q^{(m)}(B)<\infty$ and let $E_{j}^{(m)}$ be a minimizer of $Q_{j}^{(m)}$. Then $E_{j}^{(m)} \rightarrow B$ in $L^{1}, Q^{(m)}\left(E_{j}^{(m)}\right) \rightarrow Q^{(m)}(B)$ and $\alpha\left(E_{j}^{(m)}\right) / \alpha_{j}^{(m)} \rightarrow 1$.

Proof. Since $Q_{j}^{(m)}\left(W_{j}^{(m)}\right)=Q^{(m)}\left(W_{j}^{(m)}\right) \rightarrow Q^{(m)}(B)<\infty$, we can suppose that there exists a constant $\Lambda_{m}>0$ such that $Q_{j}^{(m)}\left(W_{j}^{(m)}\right) \leq \Lambda_{m}$ for all $j$. Therefore, we also have $Q_{j}^{(m)}\left(E_{j}^{(m)}\right) \leq Q_{j}^{(m)}\left(W_{j}^{(m)}\right) \leq \Lambda_{m}$ for all $j$. Again using the definition of $Q_{j}^{(m)}$ we get

$$
\Lambda_{m} \geq Q_{j}^{(m)}\left(E_{j}^{(m)}\right)=\frac{Q^{(m-1)}\left(E_{j}^{(m)}\right)-Q^{(m-1)}(B)+\left(\alpha\left(E_{j}^{(m)}\right) / \alpha_{j}^{(m)}-1\right)^{2}}{\alpha\left(E_{j}^{(m)}\right)},
$$

whence by Lemma 5.4 applied to $Q^{(m-1)}$ we obtain

$$
\Lambda_{m} \geq \frac{\lambda_{m-1}-Q^{(m-1)}(B)+\left(\alpha\left(E_{j}^{(m)}\right) / \alpha_{j}^{(m)}-1\right)^{2}}{\alpha\left(E_{j}^{(m)}\right)} .
$$


From (5.3) and thanks to the trivial estimate $\alpha\left(E_{j}^{(m)}\right)<2$ we get, for all $j$,

$$
\left(\alpha\left(E_{j}^{(m)}\right) / \alpha_{j}^{(m)}-1\right)^{2}<2 \Lambda_{m}-\lambda_{m-1}+Q^{(m-1)}(B),
$$

which means that $\left(\alpha\left(E_{j}^{(m)}\right) / \alpha_{j}^{(m)}-1\right)^{2}$ is uniformly bounded. Since $\alpha_{j}^{(m)} \rightarrow 0$ we immediately infer that $\alpha\left(E_{j}^{(m)}\right) \rightarrow 0$ as $j \rightarrow \infty$. But then the sequence $\left(E_{j}^{(m)}\right)_{j}$ converges to $B$ in $L^{1}$ and we have, by definition of $Q^{(m-1)}(B)$,

$$
Q^{(m-1)}\left(E_{j}^{(m)}\right) \geq Q^{(m-1)}(B)+o(1) .
$$

Plugging (5.4) into (5.2) we obtain after simple calculations

$$
\left(\alpha\left(E_{j}^{(m)}\right) / \alpha_{j}^{(m)}-1\right)^{2} \leq \alpha\left(E_{j}^{(m)}\right) \Lambda_{m}+o(1) \rightarrow 0 \quad \text { as } j \rightarrow \infty .
$$

We have proved that $E_{j}^{(m)} \rightarrow B$ in $L^{1}$ and $\alpha\left(E_{j}^{(m)}\right) / \alpha_{j}^{(m)} \rightarrow 1$, as $j \rightarrow \infty$. The remaining claim follows directly from the definition of $Q^{(m)}(B)$ and from the inequalities

$$
Q^{(m)}\left(E_{j}^{(m)}\right) \leq Q_{j}^{(m)}\left(E_{j}^{(m)}\right) \leq Q^{(m)}\left(W_{j}^{(m)}\right) .
$$

We now state a lemma about the $\Lambda$-minimality and the regularity of minimizers of $Q_{j}^{(m)}$ as $j \rightarrow \infty$.

Lemma 5.6 (Regularity). Let $Q^{(m)}$ be well-defined and let $Q^{(m)}(B)<\infty$. Then there exists $j_{1} \in \mathbb{N}$ such that, for all $j \geq j_{1}$ and for any minimizer $E_{j}^{(m)}$ of $Q_{j}^{(m)}$, we have

(i) $E_{j}^{(m)}$ is a $\Lambda$-minimizer of perimeter, with $\Lambda$ uniform in $j$;

(ii) $\partial E_{j}^{(m)}$ is of class $C^{1, \eta}$ for any $\eta \in(0,1)$;

(iii) $\partial E_{j}^{(m)}$ converges to $\partial B$ in the $C^{1}$-topology as $j \rightarrow \infty$.

Proof. A first attempt to prove (i) could be to directly apply Theorem 4.3. In this way, we would prove that $E:=E_{j}^{(m)}$ is a $\Lambda$-minimizer of perimeter, but we would also obtain $\Lambda=\Lambda_{j}$ dependent on $j$, and this dependence may degenerate into the (not a priori excluded) case $Q^{(m)}(B)=-\infty$. Therefore, in order to show that $\Lambda$ does not depend on $j$ we have to deal with the limit case $Q^{(m)}(B)=-\infty$, and for that we need a slight refinement of the computations already performed in the proof of Theorem 4.3.

In the following, we assume $m \geq 3$ (the case $m \leq 2$ is treated in [12]). We let $\mathcal{F}=Q_{j}^{(m)}$, that is, we set

$$
f(\alpha)=-\psi_{m}(\alpha)+\alpha^{m-1}\left(\alpha / \alpha_{j}^{(m)}-1\right)^{2} \quad \text { and } \quad g(\alpha)=\alpha^{m}
$$

in the definition of $\mathcal{F}=\mathcal{F}_{f, g}$. Then we fix a point $x \in \mathbb{R}^{n}$ and a compact variation $F$ of $E$ inside $B(x, 1 / 2)$. We distinguish the following two cases.

In the first case, we suppose that

$$
|E \triangle F|>\alpha(E) \text {. }
$$


As $\mathcal{F}(E)$ is uniformly bounded from above by some constant $C>0$, and thanks to (5.6), we obtain

$$
\begin{aligned}
\delta P(E) & \leq \psi_{m-1}(\alpha(E))+\alpha(E)^{m-1}\left(\left|Q^{(m-1)}(B)\right|+2 C\right) \\
& \leq \tilde{C} \alpha(E)<\tilde{C}|E \triangle F|
\end{aligned}
$$

with $\tilde{C}$ depending only on $Q^{(m-1)}(B), C$ and $\psi_{m-1}$. Now, from (5.7) and by the isoperimetric inequality in $\mathbb{R}^{n}$ we derive

$$
\begin{aligned}
P(E) & \leq P(B)+P(B) \tilde{C}|E \triangle F| \leq P(F)+P(B)-P\left(B_{F}\right)+C_{0}|E \triangle F| \\
& \leq P(F)+C_{1}|E \triangle F|,
\end{aligned}
$$

where the last inequality follows from Bernoulli's inequality, with a constant $C_{1}$ that does not depend on $j$.

In the second case, we suppose on the contrary that

$$
|E \triangle F| \leq \alpha(E)
$$

and observe that the constant $\Lambda$ arising in the proof of Theorem 4.3 can be estimated in a more precise way. Indeed, since by Lemma 5.5 we have $Q^{(m)}\left(E_{j}^{(m)}\right) \rightarrow Q^{(m)}(B)$ as $j \rightarrow \infty$, by the very definition of $Q^{(m)}$ and the hypothesis $Q^{(m)}(B)<\infty$, we infer that $P(E)$ is bounded by a dimensional constant and, on recalling (4.18), we get

$$
\begin{aligned}
\Lambda & =C_{n, f}+C_{n, g}|\mathcal{F}(E)| P(B)+\frac{4(n-1)}{3 n|B|} P(E) \\
& \leq C_{n}(1+\operatorname{Lip}(f)+\operatorname{Lip}(g) \cdot|\mathcal{F}(E)|),
\end{aligned}
$$

where $C_{n}$ is a positive, dimensional constant. Observe now that $\operatorname{Lip}(g)$ can be replaced by $g^{\prime}(\alpha(E))=m \alpha(E)^{m-1}$ up to possibly taking a larger constant $C_{n}$. In fact (5.9), together with Lemma 4.2 and the monotonicities of $g(\alpha)$ and of $g^{\prime}(\alpha)$, implies

$$
\begin{aligned}
|g(\alpha(F))-g(\alpha(E))| & \leq\left|g\left(\alpha(E)+c_{n}|E \triangle F|\right)-g(\alpha(E))\right| \\
& \leq c_{n} g^{\prime}\left(\left(1+c_{n}\right) \alpha(E)\right)|E \triangle F| \\
& =c_{n}\left(1+c_{n}\right)^{m-1} \cdot m \alpha(E)^{m-1} \cdot|E \triangle F|,
\end{aligned}
$$

with $c_{n}=\frac{2^{n+2}}{\left(2^{n}-1\right) \omega_{n}}$. In conclusion, we get

$$
\Lambda \leq C_{n}\left(1+\operatorname{Lip}(f)+m \alpha(E)^{m-1} \cdot|\mathcal{F}(E)|\right) .
$$

Now, to show that $\Lambda$ is uniformly bounded in $j$ we only need to estimate the product

$$
\alpha(E)^{m-1} \cdot|\mathcal{F}(E)|=\alpha\left(E_{j}^{(m)}\right)^{m-1} \cdot\left|Q_{j}^{(m)}\left(E_{j}^{(m)}\right)\right| .
$$

We first observe that the assumption $Q^{(m)}(B)<\infty$ implies that

$$
\lim _{j} Q^{(m-1)}\left(E_{j}^{(m)}\right)=Q^{(m-1)}(B) .
$$


Then we obtain the desired estimate by writing $Q_{j}^{(m)}\left(E_{j}^{(m)}\right)$ in terms of $Q^{(m-1)}$ and recalling that $m-2>0$ :

$$
\begin{aligned}
\alpha(E)^{m-1} \cdot\left|Q_{j}^{(m)}(E)\right| & =\alpha(E)^{m-2}\left(Q^{(m-1)}(E)-Q^{(m-1)}(B)+\left(\alpha(E) / \alpha_{j}^{(m)}-1\right)^{2}\right) \\
& \leq C \alpha(E)^{m-2} .
\end{aligned}
$$

Appealing again to Lemma 5.5 we see that $\alpha\left(E_{j}^{(m)}\right) \rightarrow 0$, which, by the estimate above, implies

$$
\lim _{j} \alpha\left(E_{j}^{(m)}\right)^{m-1} \cdot\left|Q_{j}^{(m)}\left(E_{j}^{(m)}\right)\right|=0 .
$$

As a result, in this case $\Lambda=\Lambda_{j} \leq C_{2}$ for some dimensional constant $C_{2}>0$. Thanks to this last estimate and to (5.8), we conclude that

$$
\Lambda=\Lambda_{j} \leq \max \left(C_{1}, C_{2}\right)
$$

which completes the proof of (i).

Finally, to prove (ii) and (iii) one can follow the proof of Lemma 3.6 in [12].

Applying Lemma 4.5 and Remark 4.6, in the following proposition we explicitly write the first variation inequality of $Q_{j}^{(m)}$ at $E_{j}^{(m)}$. Regarding the latter as a quantitative estimate of the oscillation of the weak mean curvature of $\partial E_{j}^{(m)}$, we deduce its limit as $j \rightarrow \infty$.

Lemma 5.7. Let $Q^{(m)}$ be well-defined, $Q^{(m)}(B)<\infty$ and $j_{1}$ as in Lemma 5.6. If $E_{j}^{(m)}$ minimizes $Q_{j}^{(m)}$ then, for all $j \geq j_{1}$ :

(i) $\partial E_{j}^{(m)}$ has weak mean curvature $H_{j}^{(m)} \in L^{\infty}\left(\partial E_{j}^{(m)}\right)$ (with orientation induced by the inner normal to $E_{j}^{(m)}$, and with $L^{\infty}$-norm bounded by a constant independent of $j$ ). Moreover, for $\mathcal{H}^{n-1}$-a.e. $x, y \in \partial E_{j}^{(m)}$,

$$
\left|H_{j}^{(m)}(x)-H_{j}^{(m)}(y)\right| \leq \frac{n}{n-1} \Delta_{j}^{(m)}\left(\alpha\left(E_{j}^{(m)}\right)\right),
$$

where

$$
\begin{aligned}
\Delta_{j}^{(m)}(\alpha)= & m \alpha^{m-1}\left|Q_{j}^{(m)}\left(E_{j}^{(m)}\right)\right|+\left|\psi_{m}^{\prime}(\alpha)\right|+(m-1) \alpha^{m-2}\left(\alpha / \alpha_{j}^{(m)}-1\right)^{2} \\
& +2 \frac{\alpha^{m-1}}{\alpha_{j}^{(m)}}\left|\alpha / \alpha_{j}^{(m)}-1\right| ;
\end{aligned}
$$

(ii) $\lim _{j}\left\|H_{j}^{(m)}-1\right\|_{L^{\infty}\left(\partial E_{j}^{(m)}\right)}=0$.

Proof. Note that, on choosing $f_{j}(\alpha)=\psi_{m}(\alpha)+\alpha^{m-1}\left(\alpha / \alpha_{j}^{(m)}-1\right)^{2}$ and $g(\alpha)=\alpha^{m}$, we have $Q_{j}^{(m)}=\mathcal{F}_{f_{j}, g}$. As a consequence, (i) easily follows from Lemma 4.5 and Remark 4.6, by explicitly computing the first derivatives of $f_{j}$ and $g$. Next, by Lemma 4.5, 
$\left\|H_{j}^{(m)}\right\|_{L^{\infty}\left(\partial E_{j}^{(m)}\right)} \leq 4 \Lambda /(n-1)$. Thanks to Lemma 5.5 and to the definition of $\psi_{m}$, recalling that $Q^{(1)}(B)=0$ we also have $\lim _{j} \Delta_{j}^{(m)}\left(\alpha\left(E_{j}^{(m)}\right)\right)=0$, which implies

$$
\lim _{j} \underset{x, y \in \partial E_{j}}{\operatorname{ess} \sup }\left|H_{j}(x)-H_{j}(y)\right|=0 .
$$

From this observation and arguing exactly as in [12, Lemma 3.7], one can easily complete the proof of (ii).

We finally obtain the proof of the Iterative Selection Principle:

Proof of Theorem 5.1. Statements (i) and (ii) follows from Lemma 5.5; (iii) is an elementary consequence of Lemma 5.6; and (iv) follows from Lemma 5.7 and Remark 4.6.

Acknowledgments. The research of the first author was partially supported by the European Research Council under FP7, Advanced Grant n. 226234 "Analytic Techniques for Geometric and Functional Inequalities" and by the Deutsche Forschungsgemeinschaft through the Sonderforschungsbereich 611. The second author thanks the Dipartimento di Matematica e Applicazioni "R. Caccioppoli" of the Università degli Studi di Napoli "Federico II", where he was visiting scholar during the year 2009 .

\section{References}

[1] Almgren, F. J., Jr.: Existence and regularity almost everywhere of solutions to elliptic variational problems with constraints. Mem. Amer. Math. Soc. 4, no. 165, viii+199 pp. (1976) Zbl 0327.49043 MR 0420406

[2] Alvino, A., Ferone, V., Nitsch, C.: A sharp isoperimetric inequality in the plane. J. Eur. Math. Soc. 13, 185-206 (2011) Zbl 1219.52006 MR 2735080

[3] Ambrosio, L.: Corso introduttivo alla teoria geometrica della misura ed alle superfici minime. Scuola Normale Superiore, Pisa (1997) Zbl 0977.49028 MR 1736268

[4] Ambrosio, L., Fusco, N., Pallara, D.: Functions of Bounded Variation and Free Discontinuity Problems. Oxford Math. Monogr., Clarendon Press, New York (2000) Zbl 0957.49001 MR 1857292

[5] Bonnesen, T.: Les problèmes des isopérimètres et des isépiphanes. Gauthier-Villars, Paris (1929) JFM 55.0431.08

[6] Burago, Yu. D., Zalgaller, V. A.: Geometric Inequalities. Grundlehren Math. Wiss. 285. Springer, Berlin (1988) Zbl 0633.53002 MR 0936419

[7] Campi, S.: Isoperimetric deficit and convex plane sets of maximum translative discrepancy. Geom. Dedicata 43, 71-81 (1992) Zbl 0757.52004 MR 1169365

[8] Cianchi, A., Fusco, N., Maggi, F., Pratelli, A.: The sharp Sobolev inequality in quantitative form. J. Eur. Math. Soc. 11, 1105-1139 (2009) Zbl 1185.46025 MR 2538501

[9] Cianchi, A., Fusco, N., Maggi, F., Pratelli, A.: On the isoperimetric deficit in Gauss space. Amer. J. Math. 133, 131-186 (2011) Zbl 1219.28005 MR 2752937

[10] Cianchi, A.: A quantitative Sobolev inequality in $B V$. J. Funct. Anal. 237, 466-481 (2006) Zbl 1110.46020 MR 2230346

[11] Cicalese, M., Leonardi, G. P.: On the absolute minimizers of the quantitative isoperimetric quotient in the plane. Forthcoming 
[12] Cicalese, M., Leonardi, G. P.: A selection principle for the sharp quantitative isoperimetric inequality. Arch. Ration. Mech. Anal. 206, 617-643 (2012) Zbl pre06134301 MR 2980529

[13] De Giorgi, E.: Frontiere orientate di misura minima. Editrice Tecnico Scientifica, Pisa (1961) MR 0179651

[14] Esposito, L., Fusco, N., Trombetti, C.: A quantitative version of the isoperimetric inequality: the anisotropic case. Ann. Scuola Norm. Sup. Pisa Cl. Sci. (5) 4, 619-651 (2005) Zbl 1170.52300 MR 2207737

[15] Figalli, A., Maggi, F., Pratelli, A.: A mass transportation approach to quantitative isoperimetric inequalities. Invent. Math. 182, 167-211 (2010) Zbl 1196.49033 MR 2672283

[16] Fusco, N., Maggi, F., Pratelli, A.: The sharp quantitative Sobolev inequality for functions of bounded variation. J. Funct. Anal. 244, 315-341 (2007) Zbl 1121.46029 MR 2294486

[17] Fusco, N., Maggi, F., Pratelli, A.: The sharp quantitative isoperimetric inequality. Ann. of Math. (2) 168, 941-980 (2008) Zbl 1187.52009 MR 2456887

[18] Fusco, N., Maggi, F., Pratelli, A.: Stability estimates for certain Faber-Krahn, isocapacitary and Cheeger inequalities. Ann. Scuola Norm. Sup. Pisa Cl. Sci. (5) 8, 51-71 (2009) Zbl 1176.49047 MR 2512200

[19] Fusco, N., Maggi, F., Pratelli, A.: On the isoperimetric problem with respect to a mixed Euclidean-Gaussian density. J. Funct. Anal. 260, 3678-3717 (2011) Zbl 1222.49058 MR 2781973

[20] Fusco, N., Millot, V., Morini, M.: A quantitative isoperimetric inequality for fractional perimeters. J. Funct. Anal. 261, 697-715 (2011) Zbl 1228.46030 MR 2799577

[21] Giusti, E.: Minimal Surfaces and Functions of Bounded Variation. Monogr. Math. 80, Birkhäuser, Basel (1984) Z Zbl 0545.49018 MR 0775682

[22] Hall, R. R.: A quantitative isoperimetric inequality in $n$-dimensional space. J. Reine Angew. Math. 428, 161-176 (1992) Zbl 0746.52012 MR 1166511

[23] Hall, R. R., Hayman, W. K.: A problem in the theory of subordination. J. Anal. Math. 60, 99-111 (1993) Zbl 0851.42008 MR 1253231

[24] Hall, R. R., Hayman, W. K., Weitsman, A. W.: On asymmetry and capacity. J. Anal. Math. 56, 87-123 (1991) Zbl 0747.31004 MR 1243100

[25] Maggi, F.: Some methods for studying stability in isoperimetric type problems. Bull. Amer. Math. Soc. (N.S.) 45, 367-408 (2008) Zbl 1146.49037 MR 2402947

[26] Massari, U.: Esistenza e regolarità delle ipersuperfici di curvatura media assegnata in $R^{n}$. Arch. Ration. Mech. Anal. 55, 357-382 (1974) Zbl 0305.49047 MR 0355766

[27] Tamanini, I.: Boundaries of Caccioppoli sets with Hölder-continuous normal vector. J. Reine Angew. Math. 334, 27-39 (1982) Zbl 0479.49028 MR 0667448

[28] Tamanini, I.: Regularity results for almost minimal oriented hypersurfaces in $\mathbb{R}^{n}$. Quaderni del Dipartimento di Matematica dell'Università di Lecce 1, 92 pp. (1984) Zbl 1191.35007 